\title{
Die technologische Abhängigkeit der Sowjetunion vom Weltmarkt
}

\author{
Bericht und Kommentar zu einer empirischen Studie von A.C. Sutton (1)
}

\section{Willfried Spohn}

\section{Einleitung}

Es vergeht in jüngster Zeit fast keine Woche, in der nicht in den Zeitungen über das neueste Geschäft im Ost-West-Handel berichtet würde. Die Errichtung des TogliattiWerks in Wolgograd 1968 durch Fiat, umrüstbar auf Panzer und auf dem Höhepunkt des Vietnamkriegs, schien den Anfang zu machen. Dann gab es Schlagzeilen über das erste Röhren-Erdgas-Geschäft 1970 zwischen Mannesmann und der Sowjetunion mit einem Anfangskredit von 1,2 Mrd. DM. Weniger bekannt, wurde zur gleichen Zeit die Lieferung von 1,1 Mill. t Stahlröhren im Wert von 250 Mill. Dollar gegen Erdgas mit Finsider (Italien) vereinbart (Press Reviev, 8.8.70). Dann kam es 1972 schon zum zweiten Röhren-Erdgasabkommen zwischen der SU und der BRD, wieder mit einem Anfangskredit von 1,2 Mrd. DM. Die riesigen Getreideeinkäufe der Sowjetunion, allein von den USA in Höhe von 750 Mill. Dollar, aber auch von Frankreich, Kanada und Argentinien, führten zu wilden Spekulationen über die Unproduktivität der sowjetischen Landwirtschaft. (NZZ, 10.7.72)

Doch dies war offenkundig erst der Anfang einer sprunghaft ausgedehnten Ost-West-Kooperation. Im Chemiesektor werden mit verschiedenen westlichen Firmen Großprojekte abgeschlossen. Montedison (Italien) soll chemische Betriebe im Gesamtwert von 600 Mill. Dollar in der SU bauen und dafür mit Ammoniak und anderen Chemieprodukten bezahlt werden (Neues Deutschland, 13.11.73). Zwei amerikanische Firmen sollen einen petrochemischen Komplex im Wert von 100 Mill. Dollar errichten, ebenso französische Firmen über 1 Mrd. Francs Gesamtkosten (NZZ, Handelsblatt, 11.12.73). Mit ENI (Italien) sollen im Rahmen des italienisch-sowjetischen Ölabkommens sechs petrochemische Fabriken errichtet werden, der Gesamtwert beträgt 957 Mill. Dollar und die Rückzahlung erfolgt in Rohöl, Erdgas und Produkten der petrochemischen Werke (Press Reviev, 14.5.74). Ferner wird mit der französischen Pechiney-Union-Kuhlmann-Gruppe der Bau eines Komplexes für die Gewinnung von 1 Mill. $t$ Tonerde und 0,5 Mill. t Aluminium mit einem Gesamtwert von 3,2 Mrd. DM vereinbart (NZZ, 9.12.74).

Doch auch in anderen Bereichen gewinnt die friedliche Koexistenz ökonomisch an Gestalt. Mit dem Rüstungskonzern Lockheed (USA) wird ein Vertrag über den Bau von Jumbo-Jets und Hubschraubern, sowie über Nachrichtenübermittlungsund Navigationssysteme abgeschlossen (FAZ, 15.2.74). Mit Control Data (USA)

A.C. Sutton: Western Technology And Soviet Economic Development 1917-65, 3 Bände, Stanford 1968, 1971, 1973 
wird der Kauf von Computertechnologien im Wert von 500 Mill. Dollar vereinbart (Wirtschaft des Ostblocks, 30.10.73). Durch Industrial Nucleonics (USA) sind Lieferungen von Automatisierungs- und Steuerungssystemen für die Produktion von Zellstoff und Papier, von Stahl, Gummi und Kunststoffen über 250 Mill. Dollar vorgesehen (Wirtschaft des Ostblocks, 29.6.74). Das Stanford Research Institute liefert sogar eine Laser-Anlage (Wirtschaftswoche, 31.1.75). In den Verhandlungen über eines der weltgrößten Produktionskombinate für Kunstharze mit PPG Industries, Du Pont de Nemours, Union Carbide und Amoco Chemicals (alle USA) sagt ein sowjetischer Minister ganz offen, daß bisher 150 Chemiekonzerne insb. von Westdeutschland und Großbritannien gebaut worden sind, was 7\% der sowjetischen Kapazität entspreche (FAZ, 21.3.74).

Der „Griff nach Sibirien" (Der Spiegel, 15.1.73) ist nicht nur eine groß aufgebauschte Titelgeschichte, sondern er hat in der Tat schon begonnen. Japan hat sich für 20 Jahre die Kohlevorkommen in Jakutien mit einem ersten Kredit in Höhe von 450 Mill. Dollar gesichert (Handelsblatt, FAZ, 5.6.74). Auch ein Abkommen über den Holzabbau im Umfang von 18,4 Mill. cbm Nutzholz gegen einen Anfangsk redit von 550 Mill. Dollar ist schon abgeschlossen (NZZ, 2.8.74). Da solche Großprojekte die Wirtschaftskapazität Japans offenkundig übersteigen und deshalb sich die Verhandlungen über entsprechende Erdölabkommen und den Bau einer zweiten transsibirischen Eisenbahn in die Länge ziehen (FAZ.8.4.74), springt zunehmend auch amerikanisches Kapital ein. Für die geplante Eisenbahntrasse sind mit International Harvester (USA) schon die Lieferung von Baumaschinen im Wert von 100 Mill. Dollar vereinbart (Handelsblatt, 24.1.75). Erdölabkommen für den Sachalinschelf existieren mit Japan und den USA (Wirtschaft des Ostblocks, 6.2.74 und NZZ, 29.1.75), zudem ein sowjetisch-japanisches Abkommen über Erdölsuche in Sibirien (Ostinformationen, 7.2.75). Aber das ist wohl alles nur der Anfang für die „Erschließung“ Sibiriens, die auf die astronomische Finanzierungssumme von $100 \mathrm{Mrd}$. Dollar geschätzt wird (FAZ, 27.1.75).

Anfang 1974 ist das Abkommen über das Kursker Stahlwerk mit Korf, Krupp und der Salzgitter AG perfekt. Die erste Baustufe kostet 2,5 Mrd. DM und wird von der Sowjetunion gleich bar bezahlt, insgesamt sollen 5-6 Mrd. DM aufgebracht werden (NZZ, 24.3.74 und Handelsblatt, 25.3.74). Ein zweites ähnliches Großprojekt mit den gleichen Firmen ist schon an der Ostseeküste geplant. Hierdurch sollen 5 Mill. t Eisenschwamm produziert werden, hauptsächlich gedacht für den Export nach Europa (FAZ, 19.10.74). Im Sommer 1974 kommt es schließlich zu einem dritten deutsch-sowjetischen Röhren-Erdgasvertrag. Diesmal wird gleich bis zum Jahr 2000 geplant, und gegen einen Anfangskredit von 1,7 Mrd. DM sollen jährlich 2,5 Mrd. cbm Erdgas in die BRD fließen (NZZ, 30.10.74).

Der Bau des größten Lkw-Werks der Welt an der Kama hat schon begonnen. Wenn auch Mercedes-Benz den Großauftrag von $4 \mathrm{Mrd}$. DM nicht übernommen hat, so haben die Klöckner-Humboldt-Deutz-Werke aufgrund der Verzögerung des Baus 
schon für $1 \mathrm{Mrd}$. DM Lkw's in die Sowjetunion geschickt, und 47 deutsche Firmen unter Führung von Liebherr lieferten Zubehörteile ebenfalls in Höhe von 1 Mrd. DM (Wirtschaftswoche, 24.10.74). Mehr als 100 US-Firmen sind mit 925 Mill. DM beteiligt (Wirtschaftswoche, 31.1.75). Weitere gigantische Pläne bestehen: Renault soll eine Fabrik für Lkw's und Pkw's mit der gleichen Produktionskapazität wie an der Kama - 150000 Lkw's jährlich - errichten (Handelsblatt, 10.4.72), und mit General Motors wird über ein ähnlich großes Projekt für den Bau von Nutzfahrzeugen in Sibirien verhandelt (Handelsblatt, Business International, Ostwirtschaftsreport, 8.3.74). Als vorläufiger Höhepunkt dieser gigantischen Handelsverträge kann das $20 \mathrm{Mrd}$. Dollar-Chemieabkommen mit der Occidental Petroleum Corporation (USA) gelten, das über 20 Jahre laufen soll und die Lieferung von Maschinen für Lagerung und Transport von flüssigem Ammoniak und Karbonid, sowie den Ausbau der Häfen von Odessa und Windau vorsieht. Als Gegenlieferungen sind chemische Grundstoffe wie Ammoniak, Karbonid und Chlorkalien vorgesehen. Nicht weniger überraschend ist schließlich die Lieferung eines Kernkraftwerks durch Siemens an die Memel über insgesamt 1 Mrd. DM Bausumme - wenn auch letztlich noch nicht alle Schwierigkeiten mit der DDR und Polen aus dem Weg geräumt sind -, wobei die Sowjetunion an weiteren solchen Reaktoren offensichtlich Interesse hat (FAZ, 18.7.74) und auch in der Urangewinnung an ein sowjetisch-japanisches Abkommen gedacht wird (NZZ, 10.1.74).

Soweit die möglicherweise unvollständige Geschichte der größsten Handelsabkommen zwischen der Sowjetunion und den hochentwickelten kapitalistischen Industrienationen in den letzten Jahren. Aber es gibt hunderte von weiteren kleineren Transaktionen, die von Maschinen und Industrieausrüstungen - insb. im Maschinenbau, im Chemiesektor, im elektroindustriellen und computertechnologischen Bereich - über industrielle Verfahren in der Nahrungsmittelverarbeitung, zur Herstellung von Medikamenten, Fertighäusern und Schreibmaschinen bis hin zum Import von Rohstoffen und Nahrungsmitteln reichen.

Aus allem geht hervor, daß die Sowjetunion offensichtlich einen außerordentlichen Bedarf an hochentwickelten Technologien in fast allen Produktionsbereichen hat und umgekehrt dafür hauptsächlich mit Rohstoffen und Halbfertigprodukten wie: Erdgas, Erdöl, Elektrizität, Erze und Metalle, Holz, chemische Grundstoffe, Gold, Felle, etc. zurückzahlt. Gerade die sog. Erdölkrise kam der Sowjetunion äußerst gelegen: trotz der formellen Unterstützung des arabischen Ölboykotts gegen die USA lieferte sie selbst an die EG und die USA und steigerte ihre öleinnahmen im ersten Halbjahr 1974 von 2,4 auf 7,8 Mrd. Dollar (FAZ, 22.6.74). AuRerdem fällt auf, daß zunehmend einige Fertigprodukte auf den westlichen Märkten erscheinen, darunter Werkzeugmaschinen, Autos und Flugzeuge. Ein Blick auf die Struktur des sowjetischen Außenhandels nach dem 2. Weltkrieg bestätigt diese Aussagen (2).

Betrachtet man das Außenhandelsvolumen, so stiegen die Einfuhren von 1,5 Mrd. Dollar (1950) auf 12,5 Mrd. Dollar (1971) und die Ausfuhren im gleichen Zeirraum von 1,8 Mrd. Dollar auf 13,8 Mrd. Dollar, wobei der Anteil der wesclichen Industrieländer von 15 auf $22,5 \%$ anwuchs und hierbei in den letzten Jahren die BRD, Japan, USA und 
Kommen wir endlich zur entscheidenden Frage: wie ist diese jüngste Entwicklung einer rasanten Ausdehnung des sowjetischen Außenhandels und damit der unvermeidlich verstärkte Einfluß des Weltmarkts auf die Sowjetunion zu erklären? Wie kommt es, daß nach so langer Zeit wirtschaftlicher Autarkie, plötzlich ein derartiger Umschwung eintritt? Ist diese ganze Entwicklung nur ein Zeichen dafür, daß der "Sozialismus" nun endgültig - wie schon lange angekündigt - den „Kapitalismus“ überrundet? Doch klingt das wenig überzeugend angesichts der Tatsache, daß gerade der sowjetische Außenhandel der Struktur nach mehr den Charakter eines unterentwickelten Landes hat, also hochentwickelte Technologien gegen Rohstoffe und Halbprodukte bezogen werden. Ist es vielleicht doch der grundsätzliche „Revisionismus“ der Sowjetführung, die Zielsetzungen einer ,,roten Boirgeoisie", die nach der Ausweitung des Markts im innern nun auch die wel tmarktmäßige Integration vorantreibt? Kurz: was für Ursachen haben diese jüngsten Tendenzen?

Schaut man sich hierzu die offizielle Interpretation der Sowjetunion im Lehrbuch der Politischen Ökonomie-Sozialismus an, so ist diese Entwicklung überhaupt kein Problem. Hier heißt es nur ganz lapidar, daß die Wirtschaftsbeziehungen zwischen den beiden Weltsystemen notwendig seien, daß der Handel zwischen kapitalistischen und sozialistischen Ländern im Rahmen der friedlichen Koexistenz für beide Teile nützlich sei und daß der Sozialismus den Kapitalismus ohnehin aufgrund der hohen Wachstumsraten notwendig bald erreicht und überholt haben dürfte (3). Also notwendig und nützlich, darin erschöpft sich die Erklärung. Aber auch die marxistischen Kritiker, wenn sie sich überhaupt dazu äußern, haben nur wenig anzubieten. So hält z.B. E. Mandel den Außenhandel sozialistischer Länder bei höherer Arbeitsproduktivität für einen echten Gewinn, da auf diese Weise der kapitalistische Weltmarkt gezwungen werde, durch den Handel zur sozialistischen Akkumulation in den Ländern mit einer sozialisierten ökonomischen Basis beizutragen (4). Zwischen sozialistischen und kapitalistischen Ländern scheint ein ungleicher Tausch offenkundig gerechtfertigt zu sein, aber das Problem ist ja, daß die jüngsten Technologietransfers einen umgekehrten Werttransfer in die hochentwickelten kapitalistischen Nationen einschließen. Oder A.Carlo, der die Sowjet-

Finnland die wichtigsten Handelspartner waren. Dabei stieg die Exportquote, also der Anteil der Exporte am Nationalprodukt von 4,3\% (1950) auf 6,3\% (1971), und betrug 1972 und 73 sogar $8 \%$. Der BRD-Anteil am sowjetischen Außenhandel soll 1975 sogar über $10 \mathrm{Mrd}$. DM liegen, was einer Verfünffachung seit 1970 gleichkommt. Bei den Exporten führen anteilmäßig am Gesamtexport Maschinen, Ausrüstungen und Transportmittel - hauptsächlich einfachere Typen in andere sozialistische oder unterentwickelte Länder - mit $21 \%$, es folgen Metallerze und Metallprodukte mit 18,7\%, dann Brennstoffe und Elektroenergie mit 18\%, Nahrungsgüter mit 9,2\% und Holz mit 6,3\%. Bei den Importen führen mit weitem Abstand Produktionsmittel mit 34\%, dann folgen industrielle Konsumgüter mit $20,1 \%$ und Nahrungsmittel mit $15,2 \%$. Hierzu: Länderberichte Osteuropa, Sowjetunion, hrsg. v. Koordinationsausschuß deutscher Osteuropainstitute, München 1974, Teil Außenwirtschaft, S. $236 \mathrm{ff}$.

3 Lehrbuch der Politischen Ókonomie - Sozialismus, Berlin (DDR) 1972, Kap. 49 und 50

4 E. Mandel: Zur Theorie der Obergangsgesellschaft, in: Permanente Revolution, Materialien Nr. 1, S. 35 
union für ein bürokratisch-kollektivistisches System hält, führt die Ausdehnung des sowjetischen Außenhandels auf die verstärkte kapitalistische Reorientierung zurück, die im Zusammenhang mit der wachsendenEinbeziehung von Marktelementen in die staatliche Planung stehe (5). Bleibt wieder das Problem, warum diese kapitalistische Restauration durchge führt wird.

Insgesamt ist es wohl nicht zuviel gesagt, wenn man die marxistischen Positionen gegenüber dem verstärkten Außenhandel der Sowjetunion als mehr oder weniger hilflos bezeichnet. Entweder ist er historisch unvermeidlich oder er ist ein erneuter Beweis für die kapitalistische Reorientierung der Sowjetunion, oder man macht sich zwischen diesen Positionen Gedanken über richtige und falsche Außenhandelspolitik. Doch eine wirkliche Erklärung ist weit und breit nicht zu finden. Aber es ist nur die andere Seite dieser theoretischen Hilflosigkeit, daß man an dem Faktum einer verstärkten Weltmarktorientierung der Sowjetunion nicht herumkommt.

Damit tritt eine Dimension der marxistischen Diskussion über den gesellschaftlichen Charakter der Sowjetunion wieder verstärkt ins Bewußtsein, die noch in den 20er Jahren - insb. bei Lenin, Trotzki und Preobrashenskij - eine entscheidende Rolle spielte, seit der Stalinschen Industrialisierung aber nur noch sporadisch artikuliert wurde: das Verhältnis von Sowjetunion und kapitalistischem Weltmarkt. In der ,,links"-Diskussion wurde gegenüber der Vorstellung Eggerts vom ,bürokratischen Etatismus" der Sowjetunion auf die Notwendigkeit konkret-historischer Analysen aufmerksam gemacht, die sich nicht in positivistischer Manier an vorausgesetzte Kategorien bindet, unter die das historische Material nur noch subsumiert wird. U. Schmiederer vertritt die Position, daß die Sowjetunion sich als Übergangsgesellschaft ständig im Kontext der kapitalistischen Produktionsweise bewegt habe und deshalb auch in der Analyse der Sowjetunion vom Weltmarkt auszugehen sei. E. Altvater variiert diesen Ansatz, indem die Verselbständigung des Sowjetstaats nicht als bloß innergesellschaftliches Produkt, sondern als Folge der Außenkonstituierung des Sowjetstaats durch den Weltmarkt begriffen wird. Auch H. Conert stimmt diesem Aspekt zu (6). P.W. Schulze hat ebenfalls von diesem allgemeinen Verständnis ausgehend einen ersten inhaltlichen Beitrag über den Einfluß der Weltwirtschaftskrise 1929-32 auf den 1. Fünfjahresplan vorgelegt (7). Schließlich wurde von R. Dutschke (8) und B. Rabehl (9), fortgeführt von B. Rabehl, W. Spohn und U. Wolter (10) die Marxsche Vorgehensweise bei der Analyse der zaristischen Gesellschaft wiederentdeckt, die das Verhältnis von Weltmarkt und asiatischer

A. Carlo: Politische und ökonomische Struktur der UdSSR (1917-1975), Berlin 1972, S. 100

6 Die ,links"-Diskussion befindet sich in Heft 45-47, 50, 52, 53, 57, Offenbach 1973 und 74

7 P.W. Schulze: Weltmarkt und Revolution, in ders., (Hrsg.) Ubergangsgesellschaft: Herrschaftsform und Praxis am Beispiel der Sowjetunion, Frankfurt a.M. 1973

8 R. Dutschke: Versuch, Lenin auf die Füße zu stellen, Berlin 1974

9 B. Rabehl: Marx und Lenin, Berlin 1973

10 B. Rabehl, W. Spohn, U. Wolter in Prokla 11/12 und 17/18 
Produktionsweise bei der Frage nach den Chancen einer russischen Revolution ins Zentrum stellte, und das Verhältnis von Weltmarkt und Sowjetunion für die Analyse auch der gesellschaftlichen Transformation der Sowjetunion als methodischen Ausgangspunkt betont.

Bei aller Ưbereinstimmung in diesem Punkt fällt zugleich auf, daß über das wirkliche Verhältnis von Weltmarkt und Sowjetunion zwischen 1917 und 1975 kaum Vorstellungen existieren. Es wird immer wieder auf die militärische Bedrohung oder die ideologischen Einflüsse aufmerksam gemacht, bei P.W. Schulze auch der sowjetische Außenhandel bis zum 2. Weltkrieg mit einbezogen, doch allgemein herrscht das Bewußtsein vor, daß wohl unter Lenin einige Konzessionen vergeben wurden, während der. NÖP der Außenhandel noch eine gewisse Rolle spielte, spätestens aber seit der Stalinschen forcierten Industrialisierung die direkte ökonomische Beziehung zwischen Weltmarkt und Sowjetunion verschwindend war und erst jetzt überraschenderweise die Ausdehnung des Außenhandels erfolgt. Man folgt also der Vorstellung, daß doch eine mehr oder weniger autonome ökonomische Entwicklung der Sowjetunion bisher stattgefunden hat. Wenn man lediglich den relativ geringen Umfang des sowjetischen Außenhandels 1917-75 betrachtet, scheint dieses Bild auch zu stimmen. Wenn man jedocb den Inbalt des sowjetiscben Außenbandels betracbtet, also nicbt nur den ökonomiscb-wertmäßigen Umfang, sondern die gebrauchswertmäßige Qualität, ergibt sich ein völlig anderes Bild: nämlich ein über die ganze Periode binweg bober Grad tecbnologiscber Abbängigkeit der sowjetischen Industrialisierung vom Weltmarkt.

Dies ist der Gegenstand der hierzu umfangreichsten empirischen Untersuchung, die Studie des Amerikaners A.C. Sutton. Sie soll hier in ihren wichtigsten Resultaten referiert werden. Sein Untersuchungsziel ist, den Umfang der technologischen Transfers von den hochentwickelten kapitalistischen Industrienationen in die Sowjetunion und damit den Grad der Abhängigkeit der sowjetischen Industrialisierung von der kapitalistischen Weltwirtschaft empirisch zu bestimmen. Um diese Bestimmung vornehmen $\mathrm{zu}$ können, untersucht er die wichtigsten Industriebranchen der Sowjetunion auf ihre technologische Struktur hin und versucht dann den Ursprüngen dieser Technologie nachzugehen. Gelingt die Rekonstruktion der Genesis einer Technologie, kann jeweils angegeben werden, in welchem Umfang die sowjetische Technik auf eigenständigen Quellen beruht, also indogen ist, oder von ausländischen Transfers abhängig, also exogen ist.

Dabei stuitzt sich Sutton auf umfangreiches empirisches Material, insbesondere auf die Archive des amerikanischen Außenministeriums (US State Departement Decimal File), die Archive des deutschen Außenministeriums, ab 1933 auch auf die Archive des Oberkommandos der Wehrmacht, des Oberkommandos des Heeres und der Nationalsozialistischen Partei; dann stützt er sich insbesondere auf sowjetische Publikationen, einmal die Veröffentlichungen der sowjetischen Handelsvertretungen im Ausland, zum anderen auf innersowjetische ökonomische und technische Periodika (die Trotzki- und Smolenskarchive waren offenkundig hierfür nicht so ergiebig); weiterhin zieht er Medien und Informationen westlicher Firmen, sowjetische und ausländische Zeitungsberichte und Einzeldarstellungen zu Rate. 
Sutton unterteilt sein Werk in drei Perioden: 1917-30, 1930-45 und 1945-65. Diese Einteilung ist nicht willkürlich, sondern basiert auf deutlichen Veränderungen der Form der technologischen Transfers. 1917-30 gehen sie vornehmlich in Form von Konzessionen vonstatten, 1930-45 in Form von technischen Hilfsverträgen und 1945-65 weiterhin in Form dieser technischen Assistenz ausländischer Firmen, aber beschleunigt durch Reparationen und Einbeziehung der anderen osteuropäischen Länder im Rahmen der RGW. Dabei ist die Rekonstruktion der Industrie 1917-30 technologisch völlig von der im Rahmen der Konzessionen importierten Technik bestimmt, geschieht die forcierte Industrialisierung ab 1928/30 auf dieser technologischen Basis durch die Errichtung gigantischer Fabriken mit ausländischer Assistenz und der Ausnützung ihrer Kapazitäten bis 1945, und entwickelt sich insbesondere nach 1945 eine umfangreiche Kopierung und Standardisierung ausländischer Technologie mit ausländischer Hilfe. Das verblüffende Resultat der drei Bände ist, daß die Sowjetunion von Beginn an bis heute fast vollkommen von der Technologie der kapitalistischen Weltwirtschaft abhängig ist, die Eigenständigkeit der sowjetischen Industrialisierung sich im wesentlichen auf die Anpassung ausländischer Technologien auf sowjetische Bedingungen beschränkt und nur in verschwindendem Umfang, insbesondere im militärischen Sektor auf-indogener Innovation beruht. Dabei ist zu berücksichtigen, daß Sutton bei unklarer Zurechnung eines technologischen Prozesses immer zugunsten der Sowjetunion entscheidet, die Identifikation technologischer Transfers $1917-45$ aber nur zu $70 \%$, danach aufgrund der Nichtveröffentlichung geheimer Staatsarchive in weitaus geringerem Umfang gelingt. Berücksichtigt man allerdings die Komplexität technologischer Prozesse, ist der Umfang der Identifikation technologischer Transfers durch Sutton allerdings außerordentlich und selbst vorausgesetzt, daß die vollständige Darstellung der technologischen Abhängigkeit gelänge, würde sich kaum eine Revision der grundlegenden Resultate der empirischen Studien Suttons ergeben.

Noch zwei Bemerkungen. Die eine betrifft den ideologischen Standpunkt Suttons, die zweite die Relevanz der Resultate Suttons für unsere Fragestellungen. Sutton betreibt seine Studien als Friedens- und Konfliktforscher der Hoover-Institutions mit dem klaren Zweck, der Politik des amerikanischen Staates ein empirisches Fundament zu geben, die er gegenüber der Sowjetunion aufgrund seiner Resultate für schizophren hält. Diese Schizophrenie besteht für ihn darin, daß die ,freie Welt" entweder die massiven Rüstungsausgaben drastisch reduzieren sollte, da die Sowjetunion aufgrund ihrer totalen technologischen Abhängigkeit gar nicht ihr Feind ist, oder wenn sie dies - wie es die westliche Propaganda will - doch ist, sollte sie vernünftigerweise die technologischen Transfers in die Sowjetunion stoppen. Sutton verbleibt hier völlig im Rahmen der amerikanischen Politik und erkennt nicht die Ursache dieser Schizophrenie: nämlich den kapitalistischen Akkumulationsprozeß, der sich eben sowohl im Rüstungssektor wie in der Infiltration der Sowjetunion vollzieht und von dem die amerikanische Innen- und Außenpolitik abhängig ist. Doch führt der kritische Empirismus Suttons trotz aller ideologischen Beschränkungen zu einer Untersuchungsmethode, die die übliche Abstraktion vom Weltmarkt bei der Darstellung der gesellschaftlichen Verhältnisse der Sowjetunion durchbricht, 
wie sie von sowjetmarxistischer Seite, von bürgerlicher Seite und den davon abhängigen marxistischen Theoretikern seither fast ausschließlich praktiziert wurde.

Im Vordergrund der Studie Suttons steht allerdings zunächst einmal die technologische Abhängigkeit der Sowjetunion vom Weltmarkt und nicht so sehr die Analyse der ökonomischen Vermittlung dieser technologischen Transfers. Außenhandelsstatistiken, Preise, Finanzierungs- und Kreditformen etc. müssen jeweils zusätzlich in die Analyse mit einbezogen werden, um ein vollständiges Bild zu erhalten. Trotz dieser Einschränkung sind jedoch die Resultate deshalb so gewichtig, weil sie ein vorrangiges Problem von Übergangsgesellschaften thematisiert, die aufgrund ihrer gesellschaftlichen Unterentwicklung die Industrialisierung nachholen müssen und deshalb notwendig mit der hochentwickelten Technologie der westlichen Industrienationen konfrontiert werden. Solange die proletarische Revolution in hochentwickelten kapitalistischen Ländern ausbleibt, stellt sich für diese Gesellschaften das Problem in aller Schärfe, ob sie aufgrund ihrer technologischen Rückständigkeit notwendig in Abhängigkeit vom Weltmarkt geraten oder ob es doch gewisse gesellschaftliche Alternativen gibt, die den Zusammenhang von Weltmarkt und innerer gesellschaftlicher Despotie soweit mildern, daß der Übergang in seiner rationellsten und humansten Weise vollzogen wird.

\section{1. Band: $1917-30(11)$}

1. Die Formen der Konzessionen und ibre Bedeutung für die Rekonstruktion der sowjetischen Wirtschaft

Sutton unterscheidet drei Formen von Konzessionen:

a) reine Konzessionen: „A pure concession is an economic enterprise in which a foreign company enters into a contract with the host country to organize, equip, and exploit a specific opportunity, under the legal doctrin of usufruct (d.h. ohne Erwerb von Eigentumsrechten). In return for the burden of development, exploitation, and production, the foreign company receives a non-contractual surplus or profit, usually taxed by the host country" (7), also rein ausländische Kapitalinvestitionen um Kapital zu verwerten, allerdings mit sowjetischer Besteuerung und ohne letztliche Eigentumsrechte.

b) Kredit- oder Kontraktkonzessionen, identisch mit gemischten Gesellschaften: Hier ist die Kapitalbeteiligung 50:50, später 51\% sowjetisch und $49 \%$ ausländisch, also eine Mischung von ausländischem Kapital und sowjetischer Staatsbeteiligung, die sich beide in den Profit des Unternehmens teilen. „Normally the foreign company invested capital and technology or skills and the Soviets provided the opportunity and the location. Labor, both skilled and unskilled, was partly imported and profits were to be split" (8).

11 Die Seitenzahlen im folgenden beziehen sich auf den jeweiligen Band der Suttonschen Studie 
c) Technische Hilfsabkommen: Hier werden unter genau festgelegten Bedingungen ausländische Produktionsmittel und Spezialisten vom Sowjetstaat gekauft, und fungieren damit nicht mehr als Kapital für ausländische Firmen.

In der Periode 1917-30 spielen die reinen Konzessionen und die gemischten Konzessionen die wichtigste Rolle - zwei Drittel aller Industriebranchen erhalten diese Formen von Konzessionen -, während die technischen Hilfsabkommen noch von untergeordneter Bedeutung sind, obwohl 4/5 aller Industriebranchen sie in Anspruch nehmen. 95\% der sowjetischen Industrie erhalten in irgendeiner Form ausländische Hilfe. Insgesamt wurden bis 1928/29 2.670 Konzessionen von der Sowjetunion beantragt, davon bis 1925/26 330 reine und gemischte Konzessionen und bis 1929/30 134 technische Assistenzverträge erworben. Obwohl Sutton wie gesagt nur $70 \%$ aller Konzessionen seiner Meinung nach identifizieren konnte, ist in der Sowjetunion bis 1930 keine bedeutende Technologie zu finden, die nicht durch diese Konzessionen importiert wurden. „The Western contribution to Soviet production between 1917 and 1930 was total. No important process has been isolated which was not a West-to-East transfer" (340).

Die Bedeutung dieser technologischen Transfers läßt sich auch daran ermessen, daß in denjenigen Sektoren die Wachstumsraten am höchsten waren, in denen am umfangreichsten Konzessionen vergeben wurden, also für die Produktion von Kohle, Öl, Roheisen, Walzstahl und Elektrizität. Umgekehrt stagnierten diejenigen Sektoren, die kaum westliche Hilfe erhielten. Dieselbe Korrelation ist bei den wichtigsten Exportgütern festzustellen. Auch hier gilt allgemein bei öl, Fellen, Holz, Eier, Butter, Zucker als führenden Exportsektoren 1927/28, daß hier massiv Konzessionen vergeben wurden.

Die umfassende Vergabe von Konzessionen setzte mit dem Abschluß des Rapallo-Vertrages 1922 ein. Sie ergab sich daraus, daß einmal die zaristische Industrie in sehr großem Umfang von ausländischem Kapital betrieben wurde, Facharbeiter, Ingenieure und Manager größtenteils Ausländer waren und Rußland 1917 verließen, und $\mathrm{da} B$ zum anderen deshalb die sowjetischen Arbeiter und der Sowjetstaat die sehr wohl technisch intakten Betriebe nicht selbsttätig in Schwung bringen konnten. Die Vergabe von Konzessionen schien der einzige Ausweg und es ist wohl kein Zufall, daß gerade Krassin als früherer Manager von Siemens/Schuckert in Petrograd als einer der ersten ihn vorschlugen. Die Konzessionen und die damit verbundenen technologischen Transfers verteilten sich bis 1928 insbesondere auf Deutschland und an zweiter. Steile auf die USA, die anderen westeuropäischen Länder waren unbedeutender; die ausländischen Ingenieure waren meistens Deutsche. Mit der Umstellung der sowjetischen Industrie auf die modernsten technologischen Verfahren der amerikanischen Firmen ab 1928 kommen verstärkt amerikanische Ingenieure in die Sowjetunion. (Sutton vermutet, daß diese Umstellung die materialistische Erklärung für die Schachta-Affäre ist, in der fünf Deutsche wesentlich betroffen waren.) Bis 1928 wird lediglich die zaristische Industriestruktur rekonstruiert und teilweise technologisch auf die neuesten westlichen Verfahren umgerüstet.

Diese allgemeinen Aussagen basieren nun auf einem umfangreichen empiri- 
schen Material, das sich über alle Industriezweige erstreckt. Es ist nicht möglich darüber im Detail zu referieren. Deshalb werden einige besonders wichtige Industriezweige exemplarisch dargestellt und über die anderen zusammenfassend berichtet.

\section{Die Formen der Konzessionen und ihre Bedeutung für die Rekonstruktion der sowjetischen Wirtschaft}

a) Die Rekonstruktion der Kaukasischen Ölfelder (Kap. II)

Die kaukasischen Ölfelder waren in đen 70-er Jahren des 19. Jahrhunderts entdeckt worden und produzierten 1901 mehr Rohöl als die USA und mehr als die Hälfte der Weltproduktion. Sie überlebten den 1. Weltkrieg und den Bürgerkrieg ohne wesentlichen Schaden. 1922 lag die Hälfte der Felder brach, die restlichen produzierten zunehmend Wasser. Die Ursachen waren: die Reduktion der Arbeitskräfte von 40.000 auf 10.000 , der Zusammenbruch des Transportsystems und die Reduktion der Pipeline-Kapazität, keine neuen industriellen Ausrüstungen und Reparaturen, schließlich der Zusammenbruch des elektrischen Energiesystems.

Dieser extrem desolate Zustand einer einträglichen Exportindustrie fuihrte 1921/22 zum Abschluß der International Barnstall-Konzession für die Baku-Ölfelder. Barnstall importierte Ausrüstungen auf eigenes Risiko deren Kosten plus 5\% durch den Sowjetstaat, bzw. durch die dafür zuständige Organisation Azneft getragen wurden und zwar in Goldrubeln oder in Ölprodukten zu Marktpreisen. Dazu gab es für jede 7 Fuß-ölbohrung 80.000 Goldrubel und für jedes neue Bohrloch eine Tantieme von $20 \%$ des hierdurch produzierten Öls. Azneft lieferte dafür elektrische Energie, Bohrtürme, Wasser, Ton, Holz, Zement und Gebäude. Produktionssteigerungen wurden vereinbart und die Konzession lief auf 15 1/2 Jahre.

Das vereinbarte Produktionsminimum wurde schnell überschritten. Dies gelang durch die Umstellung der äußerst arbeitsintensiven zaristischen Technologie auf modernste amerikanische Verfahren. Die ausschließliche Benutzung der Schlagtechnik 1913 wurde bis 1928 fast vollkommen durch die amerikanische Rotationsbohrtechnik ersetzt, dazuhin wurde die Pumptechnik geändert und die Maschinen elektrizifiert. Durch diese Umstellung wurde nicht nur eine gewaltige Kostenreduktion (50\%) und Produktionssteigerung für den Export erreicht, durch die steigende organische Zusammensetzung wurden auch erheblich Arbeitskräfte eingespart (40\%).

Weitere solche ölkonzessionen wurcen für die Ölfelder am Kaspischen Meer, am Schwarzen Meer, in Tiflis und auf Nord-Sachalin vergeben. Die Ölraffinerien wurden ebenfalls durch ausländische Firmen gebaut. Die ölproduktion war fast ausschließlich für den Export bestimmt und stellte eine der wichtigsten Quellen der sowjetischen Industrialisierung dar. 1926/27 war die Produktion doppelt so hoch wie 1913. Die Korzessionen wurden allerdıngs längst vor dem vereinbarten Ende aufgelöst - in dem Moment, wo die Betriebe profitabel arbeiteten und der technologische Transfer vollzogen war - und der Verkauf des öls auf dem Weltmarkt zunächst durch gemischte Gesellschaften getätigt, die dann gegen 1930 fast vollständig von sowjetischen Organisationen kontrolliert wurden. 
Das Donez-Becken produzierte 191018 Mill. Tonnen Kohle und Anthrazit, von 24 Mill. Tonnen russischer Gesamtproduktion (1913: 25,3 Mill. Tonnen). 142.000 Arbeiter waren beschäftigt, der Mechanisierungsgrad äußerst gering. Nach dem Bürgerkrieg wurden nur noch 7,2 Mill. Tonnen produziert, 47\% der Schächte geschlossen, $41 \%$ an frühere Besitzer und Bauern verpachtet, die restlichen $12 \%$, einigermaßen funktionierende, Schächte verstaatlicht, wovon die Hälfte allein 78\% der sowjetischen Gesamtproduktion förderten. Die Arbeitsproduktivität betrug 1/10 der USA, 1921-23 mußte sogar Kohle aus England und USA importiert werden.

Ab 1923 wurden umfangreiche Konzessionen vergeben. Die frïhere Eigentümerin, die französische Union-Minière-Gruppe, bekam eine reine Konzession. Abgesehen von weiteren reinen Konzessionen für englische und japanische Firmen in Spitzbergen und Nord-Sachalin, wurde das Donez-Becken dann hauptsächlich mit technischen Assistenz-Verträgen (amerikanische und deutsche Firmen) wieder in Schwung gebracht. Wenn auch die Kohlenbergwerke Staatstrusts waren, wurde die Reorganisation der Produktion völlig ausländischen Spezialisten: Managern, Ingenieuren und Facharbeitern überlassen, die zunächst selbst ohne neue Maschinerie die Produktion erheblich steigerten. Mit dem verstärkten Import neuer Maschinerie wurden einige einträgliche Schächte mechanisiert, die Produktion also konzentriert und die Anzahl der Arbeitskräfte um 20\% reduziert. (Lakonisch heißt es, daß die russischen Arbeiter diesen ausländischen Elementen feindlich gegenüberstanden). Die Mechanisierung war aber selbst 1928/29 nur zu 30\% vorgenommen. 1926/27 war das Produktionsvolumen von 1913 fast wieder erreicht (24,5 Mill. Tonnen,) allerdings unter Benutzung von nur 40\% der Schächte von 1913.

Das Donez-Becken war zugleich das Zentrum der russischen. Eisenerzförderung und der Eisen- und Stahlproduktion, 1913 wurden 550 Mill. Pud Eisenerz und 1916 2,3 Milliarden Pud Gußeisen produziert; 1920 nur noch jeweils 6 Mill. Tonnen, was dem russischen Produktionsvolumen unter Peter dem Großen entspricht. 1913 waren 160 Gebläsehochöfen und 168 Martin Stahlhochöfen in Betrieb, 1920 nur 12 bzw. 8. Von 257.000 Arbeitern im Jahre 1913 waren noch 159.000 im Jahre 1920 angestellt. Angesichts dieser äußerst verzweifelten Situation wurden 1923 insbesondere technische Assistenzverträge mit französischen, 'englischen, deutschen und amerikanischen Firmen abgeschlossen, die zur Wiedereröffnung vieler Hochöfen führten. 1927 war das Vorkriegsniveau im Donez-Becken noch nicht erreicht und selbst 1929 mit 8 rekonstruierten und einer neuerbauten Fabrik wurden erst 2,5 Mill. Tonnen Roheisen produziert, im Vergleich zu 3,2 Mill. Tonnen 1913. Diese Rekonstruktion geschah auf Basis der zaristischen Technologie und versprach keine hohen Wachstumsraten. Deshalb wurden 1927, insbesondere in der Stahlproduktion, an USA-Firmen reine Konzessionen mit einer Profitrate von $10 \%$ vergeben, die die neueste amerikanische Technologie importieren sollten. Diese Magnitogorsk-Konzession lief mit 50 Mill. Dollar 1928 an, wurde 1930 mit Verträgen über insgesamt 1 Milliarde Dollar ergänzt und sollte den Schlüssel für den ersten Fünfjahres-Plan abgeben. 
Eine besondere Rolle für den Aufbau des Sozialismus sollte nach den Vorstellungen der führenden Bolschewiki die Elektrifizierung der Sowjetunion spielen. Die Elektroindustrie war in Rußland mit Hilfe ausländischer Firmen ab 1906 entstanden, konzentrierte sich auf Petrograd und Moskau, beschäftigte 60.000 Arbeiter und erlitt während des 1 . Weltkriegs und des Bürgerkrieges keinen wesentlichen Schaden. 1920 arbeiteten nur noch 4.000 Arbeiter, die Produktion war katastrophal gering. „Many skilled Soviet workers had entered military service to get food and shelter; the more skilled foreign workers had returned home ..."(185). Ohne Konzession war dieser Situation nicht beizukommen. Es wurden vier Trusts, verbunden mit der Rückkehr früherer Manager gebildet, Verhandlungen mit General Electric und AEG geführt und mit niedrigen Löhnen und guter technischer Ausrüstung gelockt. Mit einigen reinen Konzessionen und mehreren technischen Assistenzverträgen ab 1923 wurde ein gewisser Fortschritt erzielt; allerdings um den Preis, daß die Kommunisten nur noch nominell Fabrikdirektoren waren, während die eigentlich entscheidenden Manager die weißen Direktoren, meist Ausländer, waren, und jede Fabrik zunächst auf Basis reiner Kapitalverwertung betrieben wurde. Hatte die Elektroindustrie 1913 einen Produktionsausstoß im Wert von 45 Mill. Rubel, so 1924/25: 75 Mill. Rubel und 1930: 200 Mill. Rubel. Dieses Ergebnis war jedoch himmelweit entfernt von den ersten hochfliegenden GOELRO-Plänen. Dennoch war mit Hilfe der Konzessionen die Stark- und Schwachstromtechnologie (Dammbau, Generatoren- und Turbinenbau, Transformatoren, Produktion von Elektromaschinen. Rundfunkgeräten, Telefonen etc.) auf den neuesten internationalen Stand gebracht und löste die Vorkriegstechnik völlig ab. Aber erst die umfangreichen technischen Hilfsabkommen ab 1928 mit General Electric und 1930 mit Metropolitan Vickers und AEG bereiteten das massive Wachstum in den 30-er Jahren vor.

Eines der wichtigsten Ziele, um für die Importe von Industriegïtern an harte Devisen zu kommen, war, die Position als Getreideexporteur vor dem 1. Weltkrieg wiederzuerlangen. Dies gelang nicht; im Gegenteil waren sogar 1928/29 Getreideimporte notwendig. Ab 1924 wurden deshalb zwischen den großen Staatsfarmen und Krupp (auch ein alter Bekannter im Zarenreich) reine Konzessionsverträge abgeschlossen, die sich jedoch nicht nur auf Getreideproduktion, sondern auch auf Viehwirtschaft, Zuckerrübenbau und Seidenwürmerzucht erstreckten. Aufgrund der Verluste endete man schließlich bei der Schafszucht. Aber nicht nur in diesen Bereichen wurden Konzessionen verteilt. Wichtig waren sie insbesondere in der Verarbeitung animalischer Produkte: Schinken, Butter und Eier - diese waren Konzessionen für den Export mit einem garantierten Minimalprofit von 10\% - aber auch für die Baumwollbewässerung in Turkestan wurden Konzessionen vergeben. 
Interessant ist, daß diejenigen Konzessionen, die die Anwendung von Traktoren verlangten, bald in Schwierigkeiten gerieten, da tierische und menschliche Kraft wesentlich billiger waren. Die von Sympatisanten der Sowjetunion errichteten landwirtschaftlichen Kommunen waren aufgrund von Steuern und Diebstahl allgemein ein Fehlschlag.

Die landwirtschaftliche Handwerkszeug- und Maschinenindustrie war zwischen 1905 und 1913 auf den neuesten technischen Stand gebracht worden. Diese Fabriken überlebten den ersten Weltkrieg und den Bürgerkrieg unbeschadet. 1923 wurden jedoch nur noch 12\% der Pflüge, 70\% der Sensen, 26\% der Sicheln und 5\% der anderen Ausrïstungen hergestellt. Die Bauern gingen deshalb weitgehend wieder zu Holzgerät über. Diese Zweige vernachlässigte der Sowjetstaat jedoch weiterhin, er versuchte vielmehr die Misere mit dem Bau eines sowjetischen Traktors zu beheben. Zwischen 1922 und 1926 wurden einige Modelle auch für militärische Zwecke konstruiert, doch waren die ausländischen Traktoren weit billiger und qualitativ besser. Zunächst wurden diese besteuert, um den sowjetischen Traktorbau voranzubringen, doch mußten 1925/26 dann doch fast 100\% der Traktoren importiert werden. Es war offenkundig, daß die zwei aus der Zarenzeit stammenden Betriebe: International Harvester und Putilovets die Maschinisierung der sowjetischen Landwirtschaft nicht voranbringen konnten. Ford war ab 1925 Hauptlieferant von Traktoren (von 24.000 Traktoren waren allein 20.000 von Ford) und der Sowjetstaat begann ab 1928 mit Konzessionen die ehemals zaristischen Betriebe umzurüsten und den Bau riesiger Traktorenfabriken vorzubereiten.

\section{e) Übersicht über andere wichtige Zweige}

In der NE-Metallproduktion wie Zink, Blei, Silber, Gold, Platin, Magnesium etc. wurden ab 1923 in größtem Umfang Konzessionen, meistens reine Konzessionen und einige technische Hilfsabkommen abgeschlossen. Diese Branchen waren sowohl aus militärischen wie exportmäßigen (z.B. Magnesium $=52 \%$ der Weltproduktion 1913) Gründen äußerst wichtig und konnten, da sie vor der Revolution ausländischen Firmen gehörten, ohne sie nicht wieder in Gang gesetzt werden. Ab 1928 wurden diese Zweige auf modernste amerikanische Technologie umgestellt (Kap. VI und VII).

Die sowjetische Maschinenbauindustrie, insbesondere in Leningrad und Moskau angesiedelt, produzierte 1920/21 fast nichts mehr. Deshalb mußten zunächst Maschinen importiert werden; Lokomotiven z.B. wurden im Ausland repariert und dazuhin in großem Umfang im Ausland gekauft. Obwohl die sowjetischen Werke in gutem Zustand waren, bekamen sie bis 1924 keine nennenswerten Aufträge, da keine qualifizierten Facharbeiter und Manager vorhanden waren. Erst ab 1924/25 wurden die Lokomotivbaufabriken insbesondere mit deutscher Hilfe wieder in Schwung gebracht. Ähnlich war die Situation im Dieselmotorenbau, im Werkzeugmaschinenbau, im Dampfkesselbau, in der Kugellagerproduktion und in der Präzisionsmaschinentechnologie. Sie wurden mit Konzessionen wieder rekon- 
struiert und dann ab 1928/30 auf modernste amerikanische Technologie umgerüstet (Kap. X).

Die chemiscbe Produktion (Produktion von Explosivstoffen, Dünger, Gas, Farben) lag bis 1925 weitgehend darnieder und wurde allmählich auf ausländische Verfahren umgestellt (Kap. XII).

Da Fischerei, Jagd und die dafür notwendige Konservenproduktion wichtige zaristische Exportzweige waren, wurden sie als Konzessionen vergeben (Kap. VIII). Dasselbe galt für die Holzproduktion, auch wenn dadurch vielen Bauern ihre Produktionsbasis geraubt wurde (Kap. IX). Konzessionen wurden auch für die Textilbranche (zunächst Importe von Textilmaschinen, dann Eigenbau), für den Wohnungsbau und die Nahrungsmittelverarbeitung vergeben (Kap. XIII). Die Autoproduktion kommt bis 1929 praktisch nicht voran (Kap. XIV).

Wichtig ist die deutsch-russische Zusammenarbeit im Militärsektor. Als Folge des Versailler Vertrages verlegte Junckers seine Flugzeugindustrie in die UdSSR, die dort 1923 wieder eröffnet wurde. Später wurden deutsche und amerikanische Flugzeuge gekauft, sodaß 1929 die Rote Luftwaffe ausschließlich auf ausländischer Technologie beruhte. Dazu kam die Errichtung deutscher militärischer Trainingszentren. Die Herstellung von Giftgasen, Granaten, Artillerie, U-Booten wurde mit massiver deutscher Hilfe vorangetrieben, von der die Rote Armee technologisch entscheidend abhängig war (Kap. XV).

Schließlich ist noch erwähnenswert, daß die sowjetischen Handelskompanien in Form von gemischten Gesellschaften für alle Länder gegründet wurden. Ohne sie wäre der Export von sowjetischen Waren auf dem Weltmarkt nicht möglich gewesen. Ebenso setzte eine massive Kooperation zwischen Gosbank und ausländischen Banken zur Finanzierung des Außenhandels ein (Kap. XVI).

\section{2. Band: 1930-45}

\section{Die forcierte Industrialisierung}

Die zwischen 1917 und 1930 vorherrschende Form der reinen und gemischten Konzessionen wurde nach der Rekonstruktion der sowjetischen Wirtschaft 1928/30 völlig aufgegeben. Diese oft über 15 bis 30 Jahre vergebenen Konzessionen wurden meistens durch Druck des Sowjetstaates in einer Welle der „zweiten Expropriation“ vertraglich gelöst und durch die verschiedensten Formen von technischen Assistenzverträgen ersetzt, die bis heute die vorherrschende Art des Kontrakts mit westlichen Firmen darstellt. Sie hatten meist die Lieferungen von Maschinen, Erfindungen, technischem know-how, ja ganze Fabriken, also den Import von Produktionsmitteln im weitesten Sinne zum Inhalt. Dazu wurden die damit vertrauten Ingenieure und Facharbeiter über längere Zeit gekauft, die dann jeweils an entscheidender Betriebsstelle die Produktion organisierten. Umgekehrt wurden auch sowjetische Ingenieure zur Qualifikation ins Ausland geschickt.

Die forcierte Industrialisierung ging nun in der Weise vor sich, daß der Sowjet- 
staat, bzw. Gosplan ganz allgemein über die wünschenswerte Ausdehnung bestimmter Branchen (insbesondere Kohle, Stahl, Traktorenbau) entschied und dann mit westlichen Firmen kontrahierte, um die gewünschten Technologien und Ingenieure zu importieren. Wiederum mit Hilfe westlicher Assistenz wurden schließlich die importierten Technologien den sowjetischen Produktionsbedingungen angepasst und dupliziert. Wie in der Periode 1917/30 gibt es deshalb eine ganz klare Korrelation zwischen sektoralen Wachstumsraten und dem Umfang westlicher technischer Assistenz. Die gigantischen Wachstumsraten einzelner Branchen konnten nur mit massiver Injektion westlicher Technologie erreicht werden; diejenigen Branchen, die kaum oder gar keine westliche Hilfe bekamen, stagnierten folglich.

Die forcierte Industrialisierung ging einher mit der Umstellung der sowjetischen Industrie auf die amerikanischen Verfahren der Massenproduktion. Während in der Periode vorher eindeutig die deutschen Firmen und Ingenieure den Ton angaben, orientierte sich nun die Sowjetunion an der hochentwickeltsten Technologie des neuen Demiurgs des Weltmarkts, der USA. Auch durch die Weltwirtschaftskrise und die ihr zugrunde liegende Überakkumulation gefördert, gelangte die Sowjetunion oft vor der westeuropäischen Konkurrenz der USA in den Besitz der modernsten Produktionsverfahren. Auch die Arbeitslosigkeit der amerikanischen Ingenieure im Zuge der Weltwirtschaftskrise ließ sie die Angebote der Sowjetunion leichter annehmen.

Sutton unterscheidet als materielle Grundlage der offiziellen sowjetischen Einteilung in drei Fünfjahres-Pläne und den großen patriotischen Krieg zwischen fünf klar umrissenen Abschnitten der forcierten Industrialisierung. 1926-29 beginnt die Vorbereitung des ersten Fünfjahresplans mit der Verhandlung über die Konstruktion von Fabriken mit westlichen Firmen, allerdings offiziell geheimgehalten. 1929-1932/33 werden diese Konstruktionen faktisch durch westliche Firmen durchgeführt. 1933-36/37 profitiert die Sowjetunion von der neu errichteten industriellen Kapazität - die allmählich ausgenutzt wird -, gerät aber ab 1937 aufgrund der mangelnden technologischen Transfers zwischen 1933 und 1937 in Stagnation. Diese hält an bis 1945, wenn auch die technologischen Transfers im Rahmen des deutsch-sowjetischen Nichtangriffspakts und des damit verbundenen Handelsabkommens und insbesondere im Rahmen des amerikanischen Kriegsgüterhilfsprogramms, „Lend Lease“,1941 bis 1945 die Basis für einen neuen Aufschwung legen.

Unter den Bedingungen einer solch massiven Abhängigkeit der sowjetischen Industrialisierung von der kapitalistischen Weltwirtschaft und hier insbesondere von den USA ist klar, daß der Sowjetstaat nur allgemeine Wunschvorstellungen der industriellen Entwicklung umreißen konnte, die Konkretisierung und Modifikation dieser Entwicklungspläne aber völlig von der Arbeit der westlichen Firmen abhängig war. Die "sozialistische Planung" ist deshalb auch nicht, wie der Name es will, eine durch die Gesellschaft bewußt a priori durchgeführte Planung, sondern eine projektive Rahmenplanung, die völlig von den ausländischen Kontraktfirmen - vermittelt über den Sowjetstaat - in ihrer konkreten Durchführung abhängig ist. Daraus erklären sich auch die außerordentlichen Modifikationen insbesondere des 
1. Fünfjahresplans, sei es nun die zögernde Projektion von 1928 oder seine vorschnelle Beendigung im Jahre 1932. Sutton spricht deshalb auch hart von einer „Propagandafassade“ (341).

Diest massive technologische Abhängigkeit der Sowjetunion vom Weltmarkt führt nach der Rekonstruktion der alten und der ersten Konstruktion von neuen Fabriken auch technologisch zu bestimmten Konsequenzen, falls - wie es geschieht - die Entwicklung der Produktivkräfte weiterhin auf diesen technologischen Transfers beruht. Es entsteht das Kopieren als spezifischer Entwicklungsmechanismus der sowjetischen Industrie. Produkte, Patente, technische Verfahren etc. werden - soweit möglich - gekauft und kopiert. Um dieses Kopieren überhaupt vornehmen zu können, müssen Wissenschaft, Forschungsinstitutionen und Ausbildung darauf ausgerichtet werden, die eigenständigen Forschungs- und Entwicklungskosten werden dafür eingespart.

Die durchaus noch vorhandenen, aus der zaristischen Zeit stammenden, Verfahren und Erfindungen werden - natürlich primär, weil sie unproduktiver als die westlichen sind -- ab 1930 fast völlig aufgegeben. Nur zwei Produkte: der SKB synthetische Gummi und der Ramzin-Kessel können von Sutton bis 1945 als indogene sowjetische Innovationen verbucht werden, die zudem nach 1945 wieder fallengelassen werden. Aufgrund des Kopieru-rsmechanismus wird die technologische Innovation weitgehend exogen, d.h. vo i. Entwicklung der kapitalistischen Produktivkräfte abhängig.

Sutton faßt zusammen: „Western assistance was focused by the Soviets upon single, clearcut objectives to build new, gigantic, mass-production units to manufacture large quantities of simplified standard models based on proven Western designs without design changes over a long period. Thus after the transfer of Western technology, simplification, standardization, and duplication became the operational aspects of Soviet industrial strategy" (239).

Ein weiterer Vorteil - neben der Einsparung der Forschungs- und Entwicklungskosten - bestand darin, daß in vereinfachte und standardisierte Produktionsprozesse unqualifizierte Arbeiter, wie sie die sowjetischen weitgehend waren, leichter eingegliedert werden konnten. Aber die Nachteile sind nicht zu übersehen: die sowjetischen Produktionsbedingungen sind nicht die gleichen wie in den westlichen Ländern, es besteht keine Übersicht, welche westliche Technik wirklich die entwickelste ist; die technische Initiative geht verloren und damit ist die immanente Unfähigkeit zur Innovation verbunden (,,inability hypothesis“, 317).

Im folgenden sollen diese allgemeinen Einschätzungen Suttons an einzelnen Beispielen konkretisiert werden.

2. Die technische Assistenz westlicher Firmen für die forcierte Industrialisierung

a) Eisen- und Stablin.üstrie (Kap. V)

Dieser Sektor sollte ein Eckpfeiler der Stalinschen Industrialisierung werden. Ohne 
die neuesten amerikanischen Schmelz- und Walzverfahren wäre allerdings das enorme Wachstum dieser Branche nicht möglich gewesen. Schon ab 1927 befanden sich Ingenieure der Freyn Corporation und der McKee Corporation in der Sowjetunion, um Konstruktionspläne, die den neuesten amerikanischen Roheisen- und Stahlfabriken entsprachen, zu erarbeiten und den sowjetischen Produktionsbedingungen anzupassen. Schließlich wurde die Kuznetskfabrik mit einem Output von 1 Mio. $t$ Roheisen jährlich durch den Sowjetstaat, bzw. die dafür zuständige Organisation Gipromez beschlossen. Ebenso der Bau von Magnitogorsk, dessen Kapazität ursprünglich $20 \%$ derjenigen von der United States Steel-Fabrik in Gary, Indiana, entsprechen sollte, dann aber $100 \%=2,5$ Mio. t Roheisen jährlich produzieren sollte. Allein die Planungskosten für Magnitogorsk kosteten den Sowjetstaat 2 Mio. Goldrubel. Dann wurden in riesigem Umfang Produktionsmittel und Industrieausrüstungen importiert und der Bau der Fabriken zwischen 1930 und 33 durchgefuihrt. Er lag formell in Händen von Gipromez, faktisch wurde Produktion und Planung aber von Hunderten von amerikanischen Ingenieuren überwacht und geleitet.

Nach dem ersten Bau der Fabriken, deren einfachere Teile auch mit sowjetischem Material gebaut wurden, deren zentrale maschinellen Teile (z.B. Hochöfen und Walzwerke) aber zunächst völlig importiert werden mußten, - wurden unter Assistenz der amerikanischen Firmen diese maschinellen Systeme dupliziert und auf diesem Wege die Kapazität der Werke ausgedehnt. Doch führten die besonderen sowjetischen Produktionsbedingungen, die niedrige Qualität von in der Sowjetunion selbst hergestellten Produktionsteilen, die mangelnde Qualifikation sowjetischer Arbeiter und Ingenieure für Präzisionsarbeit und nicht zuletzt der ungestüme Wille des Sowjetstaats (Stachanow-Bewegung) dazu, daß die Fertigstellung nicht in der geplanten Zeit erfolgte. In einzelnen Walzwerken war die Auslastung der Kapazität selbst 1939 nur 30\%.

Auch im Kohlenabbau wurden bis 1930/31 alle industriellen Ausrüstungen importiert. 1932/33 war der sowjetische Kohlenanbau durch den Duplikationsprozeß westlicher, insbesonderer amerikanischer Technologien selbständig, aber auch später wurden immer wieder Bergbauausrüstungen importiert. Wichtig ist auch, daß hier der Anteil der Frauenarbeit sehr hoch war (50\%) und daß zunehmend Gefangenen-Ingenieure beschäftigt wurden.

\section{b) Mascbinenbau (Kap. IX)}

Die Situation der sowjetischen Maschinenbauindustrie änderte sich 1928/30 entscheidend, als die alten Fabriken auf modernste amerikanische Technologie umgerüstet wurden. Uralmasch und Kramatorsk waren die wichtigsten neuentwickelten Fabriken zwischen 1929 und 1945 und produzierten insbesondere Maschinen für die Schwerindustrie (aber auch z.B. Unterseeboote mit deutscher Hilfe ab 1936). Trotz dieser neuen industriellen Kapazität ging jedoch der Bedarf an ausländischen Maschinen nicht zurïck. 1930 kaufte die Sowjetunion allein im Durchschnitt 40\% 
aller amerikanischen Maschinenexporte. 1931 sogar durchschnittlich 60\%. 1932 nahm die Sowjetunion von Großbritannien 90\% ihres gesamten Maschinenexports ab. 1939 wurde unter dem deutsch-russischen Nichtangriffspakt insbesondere der Import deutscher Maschinerie ausgehandelt und 1941-45 wurden unter den amerikanischen Lend Lease Program allein 1,25 Milliarden Dollar an industriellen Ausrïstungen und Maschinerie in die Sowjetunion geschickt (siehe unten).

Die Ursache für diese massive Abhängigkeit von Maschinenimporten liegt darin, daß aufgrund der allgemeinen Unterentwicklung der Produktivkräfte zunächst die allereinfachsten Werkzeuge produziert wurden, diese allerdings in massenhaftem Umfang. Bei etwas größerer technologischer Komplexität mußten Werkzeuge und Maschinerie importiert werden. Werkzeugmaschinenfabriken mußten ständig spezialisierte und automatische Ausrüstungen aus dem Ausland beschaffen, die dann mit ausländischer technischer Assistenz produzierten. In einigen Bereichen der Maschinenproduktion (Kugellager, Dieselmaschinen, Benzinmotoren) wurden zwischen 1931 und 1933 riesige Fabriken errichtet, die auf der Basis einiger weniger westlicher Prototypen in großem Umfang produzierten. Aber insgesamt war die Sowjetunion selbst 1945 über das Prototypenstadium im Maschinenbau kaum hinaus.

\section{c) Azttomobil-und Traktorenbau (Kap. XI)}

Vor 1930 wurden in der Sowjetunion nur die vorrevolutionären Fiat-Leichtlastwagen produziert, danach wurde durch massive Assistenz der Ford-Motor-Company die Amo-Fabrik in Moskau gebaut, die 50.000 Lastwagen, zusätzlich einiger großer Autos und Autobusse, jährlich produzieren sollte. Der Fiat wurde noch mit Handarbeit fabriziert. Die Amo-Fabrik basierte auf Massenproduktion. Daneben wurden noch im Laufe der 30-er Jahre zwei weitere Fabriken in Jaroslaw und Gorki gebaut, auch eine Fabrik fuir die Herstellung von Autozubehörteilen, alle mit weitgehend amerikanischer Hilfe.

Berichtet wurde über die vergeblichen Versuche bis 1926, eine selbständige Traktorenkonstruktion zu entwickeln, die ab 1926 zum Plan führten, unter technischer Assistenz von Ford ein ausländisches Modell zur Grundlage der sowjetischen Traktorenproduktion zu machen. 1929 wurde eine in den USA gebaute Traktorenfabrik demontiert, in die Sowjetunion transportiert, und in Stalingrad unter Aufsicht amerikanischer Ingenieure bis 1931 wieder aufgebaut. ,The Stalingrad Tractor Plant, therefore was American in concept, design, construction, equipment, and operation. It could just as easily have been located outside Chicago, except for the placards claiming, socialist progress" "(186). Hier wurde das International Harvester 15/30-Modell hergestellt. Zwei weitere Fabriken, die Charkow und CheljabinskTraktorenfabrik, wurden als Duplikate der Stalingrader Traktorenfabrik bis 1934 gebaut. Alle drei waren 1934 und 1941 in demselben Zustand, d.h. der vermehrte Produktionsausstoß war das Resultat wachsender Kapazitätsauslastung, die aber selbst dann noch nicht annähernd in vollem Umfang erreicht war. Interessant sind 
diese Fabriken auch deshalb, weil sie auf Panzerbau umgestellt werden konnten und faktisch auch wurden. So produzierte die Stalingrader Traktorenfabrik den T-37Tank. „The 1937 Soviet War Mobilization Plan, of which the German Wehrmacht apparently had a copy, planned to double this output in case of war" (239). Daraus schließt Sutton, daß bis 1941 nur die Hälfte der Friedensproduktion wirklich Traktorenbau, während die andere Hälfte schon Panzerbau war und folglich das sowjetische Agrarprogramm entsprechend reduzierte.

Eines der überraschendsten Resultate der Suttonschen Untersuchung ist, daß fast die gesamte industrielle Planung nicht vom Sowjetstaat selbst, sondern hauptsächlich von einem der bekanntesten amerikanischen Architekturbüros der A. KahnOrganisation durchgefuihrt wurde. „The ,outline of a program Kahn organization in 1928 was nothing less than the First and Second Five-Year Plans of ,socialist construction" “ (249).

Der Oberste Volkswirtschaftsrat der UdSSR legte Zielvorstellungen der industriellen Entwicklung vor, die Kahn in Produktionseinheiten umwandeln sollte. Allein zwischen 1928 und 1930 belief sich der Kontrakt mit der A. Kahn-Organisation auf insgesamt 2 Milliarden Dollar und bis 1939 wurden allein in den USA 0,8 Milliarden Dollar für Industriegebäude ausgegeben.

Die A. Kahn-Organisation kontrollierte und entwickelte fast alle Leicht- und Schwerindustrien, mit Ausnahme einiger militärischer Einheiten, bei denen allerdings ausländische Ingenieure, die Mitglied einer westlichen kommunistischen Partei waren, eingesetzt wurden. Eine große Verbilligung konnte hierbei durch die Standardisierung des Fabrikbaus erreicht werden. Dazu führte die A. Kahn-Organisation ein umfangreiches Management-Training durch. Entsprechend ihrer wichtigen Funktion in der Planung der sowjetischen Industrialisierung saßen amerikanische Designer und Ingenieure in allen wichtigen Organisationen des Obersten Volkswirtschaftsrates. Durch die Weltwährungskrise 1932 wurden diese technischen Hilfsverträge gestoppt, wahrscheinlich hatten inzwischen die Sowjets auch genügend über Industrieplanung gelernt und waren zudem die wichtigsten Industrieeinheiten weitgehend aufgebaut. Kahn selbst berichtet, daß die beste Industrieplanung in der GPU geleistet wurde. Sutton schätzt, daß das Verhältnis von amerikanischem und sowjetischem Produktionsvolumen in den Jahren 1928-32 durchschnittlich 6:1 jährlich war, also 9 Milliarden Dollar in den USA und 1,5 Milliarden Dollar in der Sowjetunion, und das während der tiefen Depression der Weltwirtschaftskrise. Auch hält er das faktische Produktionsvolumen zwischen 1928 und 1941 für geringer als allgemein angenommen, da die Wachstumsraten meistens Maximalziffern waren, inklusive Ausschußproduktion und niedriger Qualität. 
Die Baumwollproduktion von Turkistan hing entscheidend vom Bewässerungssystem ab. Immerhin bestanden dort seit 1825 Baumwollmanufakturen, die vor dem 1. Weltkrieg das drittgrößte Produktionsvolumen in Europa mit 8 Mill. Spindeln in 745 Manufakturen unter Beschäftigung von 388.000 Arbeitern hatten. Nach der Oktoberrevolution bis in die 20-er Jahre hinein herrschte völliger Niedergang und Stagnation vor. Schließlich wurde 1929 dem Panamakanalkonstrukteur A.P. Davis die Leitung des Baues der Bewässerungskanäle übertragen und zwischen 1928 und 1933 insgesamt 1 Milliarde Rubel investiert. Devisenmangel, Inflation und technische Schwierigkeiten ab 1931 führten allerdings dazu, daß selbst noch 1956 erst 20\% des ersten Fünfjahresplanes erfüllt waren (Kap. III).

In der NE-Metallproduktion (Kupfer, Zink, Blei, Aluminium) war die völlige technologische Abhängigkeit von westlichen Produktionsverfahren charakteristisch. Auch hier fand die Umstellung auf modernste amerikanische Verfahren zwischen 1929 und 1933 statt. Allerdings gelang aufgrund der vielfältigen Produktionsschwierigkeiten (Umstellung der amerikanischen Verfahren auf arbeitsintensive Produktion; Anwendung expropriierter Kulaken in Form von Zwangsarbeit; irrationale Antreibermethoden aufgrund der staatlich gesteckten Ziele, da diese Produktionszweige für militärische Zwecke sehr wichtig waren) die Erfüllung der gesteckten Entwicklungsziele kaum und selbst 1941 war die Kapazität vieler Fabriken noch nicht ausgelastet. Interessant ist ein Produktivitätsvergleich zwischen einem identischen Kupferschmelzer, der einmal in Kanada, das andere Mal in der Sowjetunion von der gleichen Firma gebaut wurde. In Kanada war er in 18 Monaten mit 400 Arbeitern fertig und produzierte dann 1.000 Tonnen Kupfer täglich. In der Sowjetunion wurden 5 Jahre Konstruktionszeit mit 6.000 bis 7.000 Arbeitern benötigt, und produzierte dann erst (1931) 1 Tonne täglich (Kap. IV).

Nach der Rekonstruktion und Ausdehnung der Erdölproduktion insbesondere uuf Basis deutscher Technologien 1921-30 wurde 1930-32/33 auf amerikanische Raffineriemethoden umgestellt. Die anschließend versuchte selbständige Duplikation schlug fehl, insbesondere hinsichtlich der Herstellung von Benzin mit hohen Oktanzahlen für die Luftfahrt, und führte zu technischen Assistenzverträgen bis 1945 (Kap. VI).

Die Konstruktion chemiscber Werke verlief sehr ungleichmäßig und war direkt von westlicher technischer Assistenz abhängig. Sie waren besonders wichtig für militärische Zwecke (gleiche chemische Basis von Dünger und Explosionsstoffen, Giftgasproduktion), aber selbst 1945 war kein indogenes sowjetisches Verfahren vorhanden. Zwischen 1928 und 1934 wurden die wichtigsten Chemiekombinate gebaut und die anschließende Duplikation gelang ziemlich schnell. Lediglich in der synthetischen Gummiproduktion wurde eine zaristische Technologie weitergeführt (Kap. VII und VIII).

Durch die Assistenzverträge zwischen General Electric, Metropolitan Vickers sowie AEG und dem Sowjetstaat zwischen 1927 und 1933 wurden alle wichtigen elektrotechnischen Verfahren in den Sektor der Elektroindustrie transferiert (z.B. 
Turbinenproduktion, Herstellung elektrischer Ausrüstungen oder die Konstruktion großer Kraftwerke durch ausländische Firmen) (Kap. X).

Der Ausbau und die Verbesserung des Eisenbabnsystems wurde zwischen 1933 und 1945 auf Basis amerikanischer Designs vorgenommen, insbesondere der Lokomotivbau; elektrische Lokomotiven wurden von General Electric übernommen. Hier gelang die Duplikation der Technologien ziemlich leicht und wurde unter dem Lend Lease Program 1941-45 ergänzt (Kap. XII):

Bis zum 2. Weltkrieg wurden Scbiffe auf ehemals zaristischen Werften gebaut, allerdings dreiviertel der sowjetischen Handelsmarine im Ausland. Die Rote Flotte (U-Boote, Zerstörer, Kriegsschiffe und Kreuzer) basierte zum großen Teil auf dem Kauf ausländischer Modelle, die dann mit ausländischer technischer Hilfe dupliziert wurden. Unter dem deutsch-sowjetischen Nichtangriffspakt und unter Lend Lease wurden deutsche, dann amerikanische und britische Schiffsbautechniken importiert (Kap. XIII).

Der Flugzeugbau war wiederum völlig abhängig von westlichen technologischen Transfers. Nach der Abhängigkeit von Junckers in den 20-er Jahren wurden von 1932 an extensiv ausländische Technologien erworben, insbesondere amerikanische. Schließlich wurde dieser Sektor durch das Lend Lease Program stark ausgebaut (Kap. XIV).

Für den Militärsektor faßt Sutton zusammen:, Although Soviet tanks and some guns were directly descended from Western models, a much greater degree of innovative effort was utilized on military products than in other sectors, so that the Soviets, in effect, had an indigenous military technology by $1941^{\text {" (248). Diese }}$ basierte auf den mit ausländischer Hilfe gebauten Fabriken 1930-32 und machte das Zuruickwerfen der deutschen Invasion vor dem Anlaufen des Lend LeasePrograms möglich (Kap. XV).

\section{3. Band: $1945-1965$}

\section{Das Lend Lease Program (Kap. I).}

Zwischen 1941 und 1945 wurden unter dem sogenannten Lend Lease Program insgesamt Waren im Werte von 11 Milliarden Dollar (Dollar von 1938) aus den USA, zum Teil aber auch aus Großbritannien in die Sowjetunion geschickt. (Nach FAZ vom 23.5.1972 betrugen die noch ausstehenden sowjetischen Schulden noch 800 Mill. Dollar.) Die Lieferungen umfaßten alle Art von militärischen und zivilen Gütern und lassen von daher Schlüsse zu, welche Güter die Sowjetunion aufgrund ihrer Produktionsstruktur am dringendsten benötigte. Insgesamt wurden von der US-Regierung (etwas unsystematisch) 6 Warenkategorien unterschieden. Es wurden geliefert: Flugzeuge und industrielle Flugzeugausrüstungen; Fahrzeuge insb. Tanks und LKW's, dazu alle Arten von Explosivstoffen; Lokomotiven und Schiffsausrüstungen; Nahrungsmittel; industrielle Maschinerie und Ausrüstungen; schließlich verschiedene Materialien, darunter Stahl, NE-Metalle. Telefondraht, Benzin, 
Chemikalien, Medikamente. Unter allen Kategorien rangierten insbesondere Waffen, aber auch Nahrungsmittel und Kleidung für die Rote Armee, insbesondere aber auch, was für den Wiederaufbau und den industriellen Aufschwung nach dem 2. Weltkrieg besonders wichtig ist, industrielle Ausrïstungen im Wert von 1,25 Milliarden Dollar (darunter: Motoren aller Art, Turbinen, Kompressoren, Pumpen, Kräne, Kraftstationen, elektrische Transmissionsausrüstungen, industrielle Maschinerie aller Art, auch für Nahrungsmittelverarbeitung und Textilproduktion, Gummiproduktionsanlagen, Petroleumraffinerien, Werkzeugmaschinerie, optische und wissenschaftliche Instrumente etc.).

Das Lend Lease Program wurde 1946 ergänzt durch das Pipeline-Abkommen, unter dem ebenfalls industrielle Ausrüstungen im Werte von 0,2 Milliarden Dollar in die Sowjetunion geliefert wurden, und durch das Hilfsprogramm der UNO 1945 (0,25 Milliarden Dollar an Industrieausrüstungen).

\section{Die Reparationen für die Sowjetunion nach dem 2. Weltkrieg (Kap. II)}

Primäres Ziel der Sowjetunion war, nach dem 2. Weltkrieg ein Maximum an Reparationen zu erhalten, um die vom Krieg verwüsteten Gebiete wieder aufzubauen, zugleich aber auch in den Besitz von neueren Technologien zu kommen. Insgesamt wurden von der Sowjetunion 20 Milliarden Dollar Reparationen gefordert, die sich folgendermaßen verteilten:

Deutschland

Österreich

Finnland

Italien

Rumänien

Bulgarien

Ungarn

Mandschurei
10

$$
0,4
$$

0,3

0,1

0,3

0,07

0,3

0,8
Milliarden Dollar $+1 / 3$ der deutschen Flotte
Milliarden Dollar
Milliarden Dollar
Milliarden Dollar $+1 / 3$ der
italienischen Flotte
Milliarden Dollar
Milliarden Dollar
Milliarden Dollar
Milliarden Dollar

Faktisch erhielt die Sowjetunion etwa 10 Milliarden Dollar an Reparationen, die hauptsächlich in der Demontage von Produktionsmitteln bestanden. Dabei kam der Sowjetunion in der Durchführung dieser Demontagen wahrscheinlich die Erfahrung in den Jahren 1940-42 zugute, als etwa 1.300 Industrieeinheiten hinter den Ural verlagert wurden (alle Flugzeug-, Tank- und Motorindustrien, 93 Stahlfabriken, 150 Maschinenwerkzeugfabriken, 40 Elektrofabriken).

In Deutschland stand in der Sowjetunion gemäß den Verträgen von Jalta zunächst einmal alle Fabriken der Sowjetzone und auch ein Tei? Jer Fabriken in den Westzonen als Reparationsleistungen zur Verfügung. Durch den unmittelbaren 
militärischen Ausgang des Kriegs am Tag der deutschen Kapitulation konnte aber die Sowjetunion zunächst einmal auch alle wichtigen Fabriken in ganz Berlin demontieren. Nach Übernahme der vereinbarten Besatzungsgebiete durch die Alliierten wurde in der ganzen Sowjetzone umfangreich demontiert; nachdem jedoch die Westalliierten in den ursprünglich von ihnen besetzten sowjetzonalen Teilgebieten bei wichtigen Objekten wie der Nordhausener Raketenfabrik Ingenieure und Konstruktionspapiere abgeschleppt hatten, und hier den Sowjets nur die intakten Fabrikanlagen blieben. Die Reparationen in der Sowjetzone reduzierten die dortige industrielle Kapazität in den Sektoren Fahrzeugbau, Zement-, Gummi-, Papier-, Zelluloseproduktion um durchschnittlich 60\%, dazu wurden in massenhaftem Umfang Wissenschaftler und Techniker in die Sowjetunion deportiert. Aber auch in den Westzonen wurde in großem Maßstab, unter politischem Druck auf KPD und Arbeiterschaft demontiert. Unter der sogenannten Operation RAP im Rahmen der vereinbarten Reparationszahlungen gingen $25 \%$ der in den Westzonen demontierten Industrieanlagen, und davon allein 1/3 der Reparationen in der US-Zone, an die Sowjetunion. Diese Industrieanlagen aus den Westzonen sollten gegen Lieferung von Rohmaterialien aus der Sowjetunion im Werte von $60 \%$ dieser Anlagen demontiert werden. Faktisch erhielt die Sowjetunion 50 Mill. Reichsmark an Industrieanlagen gegen 6 Mill. Reichsmark an Rohstoffen. Im einzelnen wurden in den Westzonen demontiert: Fabriken für Kugellagerproduktion, Flugzeugherstellung, Schiffsbau, Werkzeugmaschinenbau, Stahlproduktion, Herstellung von Explosivstoffen, Torpedos und Granaten, Dieselmotorenbau und Kraftstationenbau. Insgesamt für das deutsche Reparationsprogramm muß berücksichtigt werden, daß die Beschädigungen der Produktionsmittel durch die Bombardierungen in allen Zonen relativ gering war.

Auch in den anderen besetzten Gebieten wurden insbesondere Industrieanlagen demontiert. Wichtig sind hier die Demontagen amerikanischer und britischer Ölraffinerien in Rumänien, die massenhaften Demontagen japanischer Industrieanlagen in der Mandschurei, bevor die Chinesen diese Gebiete zurïckbekamen, und erwähnenswert sind die Reparationen Finnlands 1944-1955, die die Industrialisierung dieses Landes in der Produktion für die Sowjetunion entscheidend vorantrieben.

3. Übersicht über westliche Technologietransfers in wichtige sowjetische Industriezweige bis 1965

Bergbau- und Ausbubausrüstungen wie Bagger, Kräne, Bulldozer, sei es für Kohle, Erz, Torf, sind fast völlig von ausländischer Technologie abhängig (Kap. VIII).

In den NE-Metallindustrien (Nickel, Aluminium, Kupfer, Magnesium) waren die Importe sowohl durch das Lend Lease Program wie durch die Reparationen besonders groß. Doch mußten für bestimmte Produktionszwecke selbst noch 1959 z.B. 20\% der Kupferproduktion für die Herstellung von Kupferdraht importiert werden (Kap. IX). 
In der Eisen- und Stablproduktion wurden auf Basis der zwischen 1928 und 33 importierten Technologien einige sowjetische Entwicklungen vorangetrieben, insb. in der Expansion von Hochöfen. In anderen Bereichen, wo die Fortentwicklung nicht gelang, z.B. im Walzprozeß, ist das technologische Niveau immer noch auf dem US-Niveau der 30er Jahre (Kap. X).

In der Erdölindustrie sind die Ursprünge der Technologien, sei es in der Förderung von Erdöl oder in seiner Raffinierung eindeutig westlich, doch gelang es hier, einzelne indogene Entwicklungen voranzutreiben, z.B . den Turbinenbohrer. Im Raffinierprozeß war jedoch die Sowjetunion weiter von technologischen Tranfers abhängig (Lend Lease, Reparationen), und in den 60er Jahren wurden verstärkt Kontrakte mit ausländischen Firmen vereinbart, die die Produktionsverfahren modernisieren sollten (Kap. XI).

In der Chemischen Produktion gab es in den 60-er Jahren hohe Wachstumsraten in der Herstellung von chemischen Grundstoffen, wie Schwefelsäure, Chlor, etc. In den komplexeren chemischen Produktionsprozessen wie Plastikproduktion, Herstellung gemischter Düngemittel, Produktion von organisch-chemischen Stoffen, und von Pharmaka, waren die Fortschritte jedoch gering. Zwischen 1958 und 1967 wurden deshalb massive Importe von chemischen Industrieanlagen und Verfahren vorgenommen. In der synthetischen Gummiproduktion wurde nach 1945 die indogene Entwicklung des SKB aufgegeben und auf westliche Verfahren umgestellt. In der Plastikproduktion wurden alle Grundproduktionsverfahren aufgekauft, indogene Produktion liegt nicht vor (Kap. XII und XIII).

Glasproduktion, optische Industrie und Zementproduktion sind alle technologisch rückständig, basieren auf Verfahren der 30-er Jahre, die dupliziert und durch Lend Lease, Reparationen und neueste Importe aufgefrischt wurden (Kap. XIV), dasselbe gilt für Textilmaschinen und die Papierindustrie (Kap. XV).

Im Sektor der Automobil- und Traktorproduktion ist die Sowjetunion wie eh und je völlig vom kapitalistischen Weltmarkt abhängig, insbesondere von amerikanischer Technologie, aber auch deutscher, britischer und holländischer. Die zwischen 1968 und 1971 gebaute Togliatti- oder Fiatfabrik in Wolgograd hat im Wesentlichen amerikanische Technologie und ist jederzeit auf militärische Zwecke umzurüsten (Kap. XVI).

In der Herstellung von Dieselmotoren (für Schiffe und Lokomotiven) ist ebenfalls eine hohe Abhängigkeit festzustellen. 4/5 wurden im Ausland gebaut, insbesondere auch in den anderen osteuropäischen Ländern, die aber ihrerseits westliche technische Assistenz in Anspruch nehmen (das wichtigste Werk hier: die SkodaFabrik in der CSSR), die verbleibenden $1 / 5$ in der Sowjetunion gebauten Dieselmotoren waren ebenso stark auf ausländische Hilfe angewiesen (Kap. XVII).

Im Lokomotivenbau war die Basis in den 30-er Jahren gelegt worden und einige Prototypen entwickelt, aber in einzelnen Teilbereichen war auch in den 60-er Jahren die Sowjetunion auf westliche Transfers angewiesen (Kap. XIX).

Im atomaren Sektor hatte die Sowjetunion bis 1941 Pionierarbeit geleistet, dazu kam Spionage in den USA und Deutschland, so daß die Sowjetunion 1945 theoretisch das Wissen zum Atombombenbau hatte. Aber mindestens genauso 
wichtig ist die industrielle Fähigkeit, diese auch faktisch herzustellen und diese Fähigkeit kommt aus westlichen Ländern. Zentral waren hier die deutschen Reparationen, die der Sowjetunion umfangreichen Zugang zum deutschen Atomenergieprogramm verschafften, dazu wurden viele deutsche Atomphysiker bis 1955 nach Suchumi deportiert. Darauf basierten dann umfangreiche eigene Entwicklungen (auch im Atomreaktorenbau), die aber zunehmend aufgrund der Rückständigkeit im Bau von Digitalcomputern an Grenzen stößt. Sutton nimmt an, daß nicht aufgrund der theoretischen, sondern der industriellen Schwäche, die Sowjetunion stark von westlichen atomar-industriellen Verfahren abhängig war und sein wird (Kap. XVIII).

Die sowjetische Flugzeugindustrie beruht völlig auf westlicher Technologie, die insbesondere durch das Lend Lease Program und die deutschen Reparationen transferiert wurden. Diese wurde dann reproduziert und weiterentwickelt. Die sowjetische Weltraumtecbnologie stammt von der deutschen V-2-Raketentechnologie ab (Kap. XX).

Der Bau der sowjetischen Handelsmarine, die vor dem 2. Weltkrieg ziemlich unbedeutend war, wurde durch das deutsche Reparationsprogramm (sowohl Übernahme eines Teils der deutschen Flotte, wie Demontage von Werften) stark gefördert. Trotz alledem werden die sowjetischen Schiffe zum großen Teil in Finnland, Polen, der DDR und in westlichen Ländern, insbesondere in Japan und Westdeutschland (z.B. Howalt-Werke, Kiel), produziert (Kap. XXI).

1964 war der Wert der sowjetischen Werkzeugmascbinenproduktion 3/4 derjenigen der USA. Trotzdem waren und sind massive Importe in diesem Sektor zu verzeichnen. Die Antwort auf dieses Paradoxon liegt darin, daß die Sowjetunion immer wieder einige Prototypen kopiert, standardisiert und dann massenhaft produziert, aber indogene Entwicklungen, die der Vielfalt der industriellen Produktion angemessen wären, nicht aufzuweisen hat und deshalb immer wieder die neuesten Verfahren und Produkte auf dem kapitalistischen Weltmarkt aufkaufen muß (Kap. XXII).

In der elektroniscben und elektrotecbniscben Industrie ist eine massive technologische Abhängigkeit vorhanden, wo wie im Computerbau scharfe Exportrestriktionen bestanden, war die Sowjetunion weit im Rückstand. Nachdem diese 1967 gelockert wurden, setzten massive Importe ein (Kap. XXIII).

Schließlich war die Sowjetunion auch bei komplizierterer Maschinerie im Konsumgütersektor (Zuckerrübenproduktion, Nahrungsmittelverpackung, Kleidungsherstellung, Schuhproduktion z.B.) immer wieder auf westliche technische Assistenz angewiesen (Kap. XXIV).

\section{Die Entwicklungsmechanismen der sowjetischen Industrialisierung}

Die Entwicklungsmechanismen der sowjetischen Industrialisierung nach 1945 setzen die Struktur der forcierten Industrialisierung ab 1928 fort. Wichtige und kaufbare Innovationen der kapitalistischen Weltwirtschaft werden importiert (seien 
es ganze Fabriken oder einzelne Verfahren und Produkte) kopiert, standardisiert und dupliziert und dies meistens mit westlicher technischer Assistenz. Sutton schätzt, daß zu jedem Zeitpunkt bis heute über die meisten industriellen Sektoren gestreut 100 solcher technischer Assistenzverträge in Kraft waren. Da die Sowjetunion weitgehend auf exogene Innovationen zurückgreift, um eigene Forschungsund Entwicklungskosten $\mathrm{zu}$ sparen, entwickelt sie selbst nur wenige indogene Innovationen, die an einer Hand aufzuzählen sind, und diese insbesondere im militärischen Sektor, so daß sie auch in Zukunft immer wieder auf die höher entwickelten kapitalistischen Produktivkräfte angewiesen sein wird. Von daher ist klar, daß die Sowjetunion nur in den Sektoren, wo unmittelbar die neuesten westlichen Technologien erworben werden, und im Militärsektor technisch auf Weltmarktniveau ist, ansonsten ist sie - und das gilt für die meisten Bereiche - technisch rückständig: im Durchschnitt $10-30$ Jahre gegenüber den USA. „From the technical viewpoint the Soviet Union at 1970 is a copy-a rather imperfect copy- of the West ${ }^{6 /}$ (415). Diese Rückständigkeit drückt sich auch darin aus, daß die sowjetischen Exporte noch heute die typische Zusammensetzung unterentwickelter Länder aufweist, nämlich Rohmaterialien und Halbfertigwaren wie Magnesium, Chrom, Felle, Nahrungsmittel, Eisenerz, Glasblöcke etc.

Sutton schließt hieraus, daß es nicht an theoretischer Forschung mangelt, sondern an der fundamentalen Schwäche, industrielle Innovationen voranzutreiben. Diese seien aber aufgrund des zentralen Planungssystems nicht möglich, und Sutton fordert deshalb die Rückkehr zu Marktwirtschaft und Kapitalismus in ihrer westlichen Reinheit.

\section{Einige theoretische Konsequenzen}

Viele, die bisher gefolgt sind, werden sich vielleicht insgeheim sagen: der methodische Ansatz, das Verhältnis Sowjetunion-Weltmarkt zum Ausgangspunkt für die Analyse der gesellschaftlichen Entwicklung der Sowjetunion zu nehmen, ist ja ganz einsichtig, aber die referierten Ergebnisse des A.C. Sutton sind ja alles andere als glaubwürdig, im Gegenteil allzu verwandt mit Kalten-Kriegs-Parolen. Also das berühmte Stahlwerk in Magnitogorsk soll im wesentlichen amerikanische Technologie sein? Oder die Traktorenfabrik in Stalingrad soll in toto eine demontierte amerikanische Fabrik sein? Oder gar, der 1. Fünfjahresplan ist in seiner Konkretisierung das Werk amerikanischer Ingenieure?

Demgegenüber kann nur gesagt werden: Einmal ist es selbstverständlich, daß diese Resultate empirisch überprüft werden müßten, viele Ergebnisse aber durchaus mit anderen Untersuchungen übereinstimmen und aufgrund der Quellengrundlage außer- und innersowjetischer Publikationen zu keinem Zweifel Anlaß geben. Zum andern liefern die Ergebnisse - bei aller Lückenhaftigkeit im Detail - eine festere Grundlage für eine materialistische Erklärung der gesellschaftlichen Entwicklung der Sowjetunion im Kontext des kapitalistischen Weltmarkts. Während viele Einschätzungen der Sowjetunion schnell zu Begriffen wie sozialistische Basis, Über- 
gangsgesellschaft, rote Bourgeoisie, Fehler der sowjetischen Führung, Deformation des Arbeiterstaats etc. zurückgreifen, ist es auf Basis solcher Ergebnisse möglich, aus den konkreten Zusammenhängen von Weltmarkt und sowjetischer Entwicklung die Entstehung des Stalinismus, den Übergang zu den ökonomischen Reformen und zum verstärkten Ost-West-Handel plausibler zu erklären und damit von eventuell ideologischen Prämissen zu wirklich gesellschaftlichen Erklärungen zu kommen. Dies soll im folgenden thesenartig skizziert werden.

\section{Entwicklung und Unterentwicklung}

Das Phänomen der technologischen Transfers ist so alt wie die kapitalistische Produktionsweise selbst und ist im Kontext der ungleichzeitigen Entwicklung der kapitalistischen Produktionsweise zu begreifen. Kommt es in einem Zentrum zu Prozessen rascher Akkumulation des Kapitals - historisch zuerst in England mit dem Durchbruch der kapitalistischen Industrialisierung ab 1780 -, so entsteht damit das für die Geschichte der kapitalistischen Produktionsweise typische Produktivitäts- und d.h. Technologiegefälle, was sich in Profitraten- und Preisgefällen auf dem Weltmarkt äußert. Die nicht mitziehenden Gebiete werden zu relativ unterentwickelten Regionen, die nun nicht einfach die Entwicklung Englands nur etwas später und zeitlich verschoben in gleicher Weise durchlaufen, sondern in charakteristischer Form durch die Dominanz eines kapitalistischen Zentrums auf dem Weltmarkt in ihrer Entwicklung, sofern überhaupt eine stattfindet, modifiziert werden. Nur in England kann von einer originären Geschichte der kapitalistischen Produktionsweise gesprochen werden, die nicht durch andere, überlegene Zentren kapitalistischer Akkumulation beeinflußt wird, deshalb aber gerade in historisch untypischer Form verläuft. Während hier die kapitalistische Industrialisierung weitgehend ohne Staatsunterstützung und mithilfe kleinerer kommerzieller Banken organisch wachsend und mit relativ geringen Wachstumsraten vonstatten geht, verstärken sich die Symptome der Unterentwicklung, je größer der Abstand zum entwickeltsten Zentrum, damals England, wird. Unter Konkurrenzdruck stehend, ohne entsprechende interne Verwertungsmöglichkeiten, ohne die entwickelten Technologien des überlegenen Landes auf dem Weltmarkt, müssen zentralisiertes Bankkapital, Staatsunterstützung und ausländisches Kapital die fehlenden internen Ressourcen der Entwicklung ersetzen. Gelingt eine solche Industrialisierung unter unterentwickelten Bedingungen, so sind typischerweise in den entsprechenden Sektoren hohe Wachstumsraten zu verzeichnen (12).

Neben der breitgefächerten marxistischen Diskussion zur Ungieichzeitigkeit des Weltmarkts und zum ungleichen Tausch sind zum Verhäl tnis Entwicklung/Unterentwicklung in Europa besonders instruktiv die Beiträge des Wirtschaftshistorikers und Wachstumstheoretikers A. Gerschenkron: Economic Backwardness in Historical Perspective, Cambridge/Mass. 1962, Continuity in History and Other Essays, Cambridge/Mass. 1968 und Europe in the Russian Mirror, Cambridge 1970 
In den hinter England noch am weitesten entwickelten Ländern, Belgien und Frankreich, deuten sich diese Symptome nur an. Englische Unternehmer investieren direkt durch den Aufbau eigener Fabriken und transferieren damit das fehlende technische know-how selbst. In Frankreich kommt ihnen der Staat mit Prämien und Absatzsubventionen zuhilfe, in Belgien wird das notwendige Geldkapital durch Investitionsbanken zentralisiert. Spätestens ab 1830 steht die Akkumulation des Kapitals auch in diesen Ländern auf eigenen Beinen. Geht man weiter östlich, so verstärken sich die Muttermale der Unterentwicklung für die Geschichte der kapitalistischen Produktionsweise ganz erheblich. Schon in Deutschland ist der preußische Staat aufgrund des geringen Profitratenniveaus gezwungen, eine massive Industrieförderungspolitik vorzunehmen, kann der Anstoß der Industrialisierung durch den Eisenbahnbau nur über die Zentralisation des Kapitals durch Aktienbanken und Aktiengesellschaften und über Zinsgarantien des preußischen Staats geschehen. Der Einfluß des ausländischen Kapitals ist schon erheblich: Staatsanleihen werden insb. in England und Frankreich aufgebracht, ein großer Teil der Aktienbanken ist in ausländischen Händen, englische, französische und belgische Unternehmer tragen in großem Umfang zwischen 1840 und 70 - im schwerindustriellen Sektor kann er auf 25\% der deutschen Gesamtproduktion geschätzt werden - zur deutschen Industrialisierung bei. Im Laufe von 30 Jahren konnte der technologische Vorsprung Englands, Frankreichs und Belgiens durch Deutschland eingeholt und dann sogar zusammen mit den USA ab 1890 überholt werden (13).

Im Extrem kombinieren sich die Symptome der Rückständigkeit bei der Industrialisierung des zaristischen Rußland. Schon der Aufbau von staatlichen Manufakturen unter Peter dem Großen war nur auf Basis ausländischen Kapitals, in Form des Imports von Techniken und den damit vertrauten Unternehmern und Handwerkern möglich. Sie hatten als notwendiges Pendant die massive Verstärkung despotischer Formen der zaristischen Autokratie zur Voraussetzung. Die Kontrolle und Besteuerung der Bauern wurde bis zur äußersten Grenze der Belastbarkeit gesteigert, die Staatsbetriebe glichen Festungen und preßten die Bauern zwangsweise zur Arbeit. Dieses Zusammenspiel von Westorientierung und Verstärkung asiatischer Elemente, von merkantilistischen und despotischen Staatsfunktionen wiederholt sich auch bei der Industrialisierung des zaristischen Rußland ab 1870. Einmal kann die Industrialisierung nur durch den extensiven Import ausländischen Kapitals geschehen, seien es Technologien, Ingenieure und Facharbeiter, oder sei es ausländisches Geldkapital in Form von Staatsanleihen oder Portfolioinvestitionen. Zum andern ist der zaristische Staat aufgrund der weitgehend fehlenden Verwertungsmöglichkeiten des Kapitals gezwungen, in großem Umfang staatlich einzugreifen: beim Eisenbahnbau kauft und garantiert er die ausländischen Aktien, aber auch

13 Zum britischen Kapitalexport: W.O. Henderson, Britain and Industrial Europe 1750-1870, Leicester UP 1965 und L.H. Jenks, The Migration of British Capital to 1875, London 1963. Zum französischen Kapitalexport: R.E. Cameron, France and the Economic Development of Europe 1800-1914, Princeton 1961; allgemein D.S. Landes, Der entfesselte Prome theus, Köln 1973 
sonst übernimmt er gewichtige Kredit- und wichtige Absatzfunktionen. Schließlich ist er gezwungen, um diese Industrialisierung überhaupt wahrnehmen zu können, die despotisch- fronwirtschaftliche Abhängigkeit der Bauern zu befestigen, daran haben auch die Bauernbefreiungsgesetze 1861-65 nichts ändern können. Denn nur über Hungerexporte und drückende direkte und indirekte Steuern können interne Staatseinnahmen geschaffen werden. Dieser Widerspruch zwischen der durch ausländisches Kapital induzierten, hochkonzentrierten und -zentralisierten Industrie mit extensiven staatlichen Vermittlungsfunktionen und dem despotisch ausgepreßten landwirtschaftlichen Sektor, in dem sich nur einige Großgrundbesitzer und Großbauern zu modernem, weltmarktorientierten Grundeigentum mausern konnten, kennzeichnet in extremer Form eine kapitalistische Industrialisierung unter rückständigsten Bedingungen und ist die Ursache für Arbeiter- und Bauernrevolution in Rußland. Wenn auch der Staat nach 1905 die Kreditfunktionen stärker Privatbanken überließ und die Obscina gesetzlich beseitigte, also die privatkapitalistischen Elemente stärker hervortraten, so waren 1914 noch lange nicht die Bedingungen einer sich selbst tragenden Akkumulation des Kapitals auf breiter gesellschaftlicher Grundlage wie in Westeuropa gelegt. Fast 50\% des Aktienkapitals kam 1916 aus dem Ausland (insb. aus Frankreich), und das Produktivitätsniveau war fast vollkommen von ausländischen (insb. deutschen) Technologietransfers abhängig; entsprechend waren Unternehmer und Techniker oft Ausländer (14).

Man sieht, die kapitalistische Entwicklung eines Landes vollzieht sich weder autonom, noch in typischen Stadien, sondern in einem von vornherein internationalen Zusammenhang, der die kapitalistische Industrialisierung unter relativ unterentwickelten Gesellschaftsbedingungen bestimmt. Je rückständiger diese Bedingungen im Verhältnis zu den hochentwickelten Zentren des Weltmarkts sind, desto gravierender wird der Einfluß des ausländischen Kapitals und desto extensiver die staatliche Vermittlung bei der Entwicklung, sofern überhaupt eine solche Staatsmacht vorhanden ist. Im kapitalistischen Rahmen gibt es letztlich nur zwei Alternativen: entweder gelingt dem rückständigen Land über diese staatlichen Krücken eine eigenständige Akkumulation des Kapitals - wie z.B. Deutschland, Norditalien, oder auch Japan - oder die Übermacht des akkumulierten Kapitals in den entwickelten Ländern ist so groß, daß diese Form der Industrialisierung nicht mehr gelingt und nur die für das ausländische Kapital profitablen Sektoren entwickelt werden - also das Schicksal einer abhängigen und sektoralen Entwicklung, wie es die meisten der heute unterentwickelten Länder getroffen hat.

\section{Unterentwicklung und Übergangsgesellschaft}

Die gesellschaftliche Entwicklung des zaristischen Rußlandsbewegte sich offensicht- 
lich gerade zwischen diesen Alternativen. Die Widerspriiche einer kapitalistischen Industrialisierung unter Bedingungen extremer Rückständigkeit, vermittelt über die despotische Gewalt der zaristischen Autokratie, produzierten eine Doppelrevolution, die 1905/6 noch zögernd, 1917 aufgrund der Schwächung der Staatsmacht im 1. Weltkrieg erfolgreich die fronwirtschaftliche Abhängigkeit der Bauern beseitigte und in den Betriebskommitees, Gewerkschaften und Sowjets erste Formen sozialer Emanzipation freisetzte. Die Frage war, ob die Formen der sozialen Emanzipation die weitere Entwicklung der sowjetischen Gesellschaft bestimmten, oder ob umgekehrt die in den Muttermalen der russischen Gesellschaft angelegten Gegentendenzen wieder die Oberhand gewinnen würden. Die proletarische Revolution im Westen, die der russischen zuhilfe kommen sollte, blieb aus. Unter diesen Bedingungen der weltrevolutionären Isolierung, der Intervention der ausländischen Mächte, dem Ausbruch des Bürgerkriegs, dem extremen Niedergang der Produktion, waren schon in der Periode des Kriegskommunismus die Gegentendenzen der alten Gesellschaft in Form der Zwangsrequirierungen von Getreide, der Zentralisierung der Arbeiterbewegung und der mit kapitalistischen Methoden staatlich organisierten Produktion angelegt.

Um den lebensnotwendigen Wiederaufschwung der Wirtschaft in Gang zu setzen, war der Übergang zur NÖP, d.h. die staatliche Anerkennung der privat produzierenden Bauernschaft und der in kapitalistischen Formen produzierenden Betriebe unter Kontrolle des Sowjetstaats der einzige Ausweg. Die Verselbständigung des Sowjetstaats von den Formen sozialer Emanzipation konnte unter solchen Bedingungen kaum zurückgenommen werden (15). Im Gegenteil. Auf der Basis der Resultate Suttons und der Analyse des sowjetischen Außenhandels (16), ist offen-

15

16

Ausführlicher ebda., Teil III

Vergleicht man die sowjetische Außenhandelsstruktur bis zum 2. Weltkrieg mit der zaristischen, so sind 1909-13 unzweifelhaft die Getreideexporte mit 40,1\% des Gesamtexports (6,5 Mrd. Rubel) die Hauptquelle für den Import von industriellen Gütern, so beträgt der Anteil der Maschinerie am Gesamtimport 1913 (6,2 Mrd. Rubel) z.B. 11,4\%. Durch die Expropriation gerade des weltmarktorientierten Grundeigentums und die Ablösung der zaristischen Despotie über das Dorf durch Waren- und Geldbeziehungen im Gefolge der Oktoberrevolution können die Getreideexporte nicht mehr diese hervorragende Rolle spielen. $1926 / 7$ haben sie nur noch einen Anteil von fast $25 \%$ am Gesamtexport (3,4 Mrd. Rubel) und fallen dann sogar 1927/8 und 1929 fast ganz aus. Durch die Zwangsrequirierungen werden sie wieder auf fast die Hälfte der zaristischen Exportmenge 1909-13 mit einem Anteil von 19\% des Gesamtexports (4,5 Mrd. Rubel) angehoben. Der Produktionsrückgang in der Landwirtschaft und der allgemeine Preisfall für Nahrungsmittel im Gefolge der Weltwirtschaftskrise lassen die Getreideexporte bis 1937 in ihrer Bedeutung weit hinter Holz- und ölexporte zurücktreten. Umgekehrt erreichen die Importe von Maschinerie schon 1925/6 anteilmäßig und 1929 auch wertmäßig die zaristische Quote, und haben in den Jahren 1930-32 mit durchschnittlich über $30 \%$ Anteil am Gesamtimport (durchschnittlich etwa 4 Mrd. Rubel) einen eindeutigen Höhepunkt, den sie aufgrund des fallenden Exportwerts vor dem 2. Weltkrieg nicht mehr annähernd erreichen. Uber die ganze Periode hinweg besteht immer eine klare Korrelation von Produktionsmittelimporten und industriellen Wachstumsraten. Zum sowjetischen Außenhan del bis 1939 insb. A. Baykov: Soviet Foreign Trade, Princeton 1946 
kundig, daß die Rekonstruktion der Industrie nur über massive Konzessionsvergabe, den Import von Produktionsmitteln und den hierfür notwendigen Export von Nahrungsmitteln und Rohstoffen verlief. Diesen ökonomischen Tendenzen wurden schon während der NöP die emanzipatorischen Formen zunehmend staatlich untergeordnet. Dieser Weg ist nicht weiter erstaunlich, wenn man den Niedergang der Weltrevolution, die extreme Auslandsabhängigkeit der zaristischen Industrie, das Angewiesensein auf ausländische Ingenieure und Facharbeiter, sowie das allgemein niedrige Qualifikationsniveau der russischen Arbeiterschaft berücksichtigt. Aber dieser Weg reproduzierte tendenziell in neuer Form das alte Dilemma der zaristischen Gesellschaft: eine in Kooperation mit dem ausländischen Kapital stattfindende Industrialisierung, die kaum auf die Bedürfnisse der Landwirtschaft Rücksicht nahm, und die auf der Abschöpfung des Mehrprodukts der Bauernschaft beruhte. Die NÖP ist noch nicht die faktische Wiederherstellung dieser alten Struktur, sie ist ein labiles Gleichgewicht, wo die sozialen Ansprüche des Proletariats und die bürgerlichen Ansprüche der Bauernschaft den Muttermalen der zaristischen Gesellschaft noch die Waage halten. Aber die Disproportionen von Landwirtschaft und Industrie, die sich in den Scherenkrisen äußern, die Abhängigkeit des Tempos der Industrialisienung von dem nur in Geldsteuern abschöpfbaren Mehrprodukt der Bauernschaft fordern eine Lösung.

Als die Bauern aufgrund der gesellschaftlichen Disproportionen kein Getreide mehr ablieferten, da sie dafür nichts Gleichweritges kaufen konnten, dem Sowjetstaat die Herstellung der zaristischen Exportstruktur nicht gelang, im Gegenteil, die laufenden und in Aussicht genommenen Verträge mit westlichen Firmen und damit eine schnellere Industrialisierüng in Gefahr geriet, wurde dem Sowjetstaat die Lösung unvermeidlich aufgezwungen. Die Kollektivierung der Landwirtschaft schien an den ,sozialistischen Klassenkampf auf dem Lande“ während des Kriegskommunismus anzuknüpfen, und die forcierte Industrialisierung schien die Verwirklichung des Sozialismus zu sein. Aber in Wirklichkeit war es eine Zwangskollektivierung, die die despotische Kontrolle des Sowjetstaats über die Bauernschaft herstellte, um für die forcierte Industrialisierung die notwendigen Mittel bereitzustellen, die die Bauernschaft nicht freiwillig, d.h. über den Markt geben wollte und konnte. Aber in Wirklichkeit ist es eine Industrialisierung, die nicht emanzipatorische Formen der Arbeit zur Grundlage hat, sondern die auf das ausländische Kapital, seine Technologie und seine qualifizierten Arbeitskräfte angewiesen ist, und die umfangreich in Form der Zwangsarbeit durch die despotische Gewalt des Sowjetstaats der sowjetischen Gesellschaft aufoktroyiert wurde. Da die hochentwickelten kapitalistischen Industrieländer in der schärfsten Überakkumulakrise ihrer Geschichte steckten, kam ihnen ein Absatzmarkt für Produktionsmittel gerade gelegen. Der Stalinismus ist auf einem höheren Niveau des kapitalistischen Weltmarkts und in neuer Form die Reproduktion des alten Dilemmas einer Industrialisierung unter extrem rückständigen Bedingungen. Die anfänglichen industriellen Wachstumsraten von 12-13\% übertrafen die besten zaristischen Raten (9\%), doch sind sie auf Basis der „Vorteile ${ }^{6 /}$ der Unterentwicklung sehr wohl erklärlich (17). 17 Diese bereinigten Wachstumsraten bei A. Gerschenkron: Notes on the Rate of Industrial Growth in Soviet Russia, in: ders., Economic Backwardness in Historical Perspective, a.a.O.,S. 254 ff. 
Die staatliche Planung der ersten Fünfjahrespläne scheint bewußt-gesellschaftliche Planung zu sein, aber ihr Inhalt ist die Koordination dieser Vermittlung von modernsten kapitalistischen Produktivkräften mit despotisch-gesellschaftlichen Formen der Zwangskollektivierung und Zwangsarbeit.

In dem Maße, wie die Industrialisierung voranschreitet, die Rückständigkeit überwunden wird, die gesellschaftlichen Produktivkräfte mannigfaltiger und komplexer werden, treten auch die despotischen Formen des Staates zurïck, wird der Stalinismus liberalisiert. Die ökonomischen Reformen des Neuen Systems der Planung und der Leitung sind Reformen der Staatsplanung, die diesen entwickelteren gesellschaftlichen Bedingungen geschuldet sind. Sie können nicht mehr über despotische Formen, sondern zunehmend über das ,sozialistische Wertgesetz", über staatlich vermittelten Profit, Kredit, Preis etc. koordiniert werden, d.h. der Staat wird allmählich ins Wertgesetz zurückgenommen. Aber selbst diese Reformen sind immer noch Ausdruck einer wohl wesentlich reduzierten, aber doch relativen Rückständigkeit gegenüber dem kapitalistischen Weltmarkt. Der verstärkte Ost-West-Handel gründet auf dieser noch bestehenden Rückständigkeit, aber auch auf entwickelteren gesellschaftlichen Produktivkräften der Sowjetunion, die auf dem Weltmarkt konkurrenzfähig werden. Zudem verschärfen sich auf dem kapitalistischen Weltmarkt die Tendenzen einer erneuten allgemeinen Überakkumulationskrise, die nach erweiterten Märkten gerade für die besonders anfällige Produktionsmittelindustrien verlangt. Wird die relative Rückständigkeit in einigen wichtigen Sektoren der Sowjetwirtschaft überwunden, die Konkurrenzfähigkeit auf dem Weltmarkt und d.h. die Verwertungsmöglichkeit hergestellt, werden diese Industriesektoren das Wegwerfen der staatlichen Krücken, die Ausschaltung der staatlichen Kontrolle verlangen. Die sowjetischen Arbeiter werden darüber entscheiden, ob diese Form von „Einholen und Überholen“ eine Morgenröte eines reineren Kapitalismus oder eine Morgenröte eines erneuten Sozialismus, und d.h. die soziale Freisetzung der durch die stalinistische und nachstalinistische Industrialisierung vergesellschafteten Formen der Arbeit, sein wird. Die schon zentralisierten Formen der verstaatlichten Industrie und Landwirtschaft werden dann ökonomisch und politisch ihren Inhalt ändern und in die Gesellschaft zurïckgenommen.

\section{Schlußbemerkung zur Diskussion über den gesellschaftlichen Charakter der Sowjetunion}

Es ist hier ein Interpretationsrahmen der sowjetischen Entwicklung skizziert worden, der bewußt das Verhältnis von Weltmarkt und innerer gesellschaftlicher der Sowjetunion in den Mittelpunkt stellte, um Kontinuität und Diskontinuität der ökonomischen Bedingungen von zaristischer und sowjetischer Gesellschaft aufzuzeigen. So wie es nicht möglich ist, unter Abstraktion vom Weltmarkt die Entwicklung der zaristischen Gesellschaft aus sich heraus zu erklären, so muß auch jeder Versuch fehlschlagen, die Sowjetunion als mehr oder weniger autonome Gesellschaft zu analysieren. In diesem Kontext interessieren auch die Resultate der 
Suttonschen Untersuchung, die die immer wieder stillschweigend vorausgesetzte Prämisse der (relativen) ökonomischen Autarkie der Sowjetunion ad absurdum führt und zeigt, in welch massivem Umfang die Sowjetunion von Anfang an auf die technologischen Transfers seitens der hochentwickelten Industrienationen angewiesen war und noch in weiten Bereichen angewiesen ist, auch wenn bis zuletzt der wertmäßige Umfang des sowjetischen Außenhandels gering gewesen war.

Einige weitere solcher kategorialer Setzungen, die die Diskussion um den gesellschaftlichen Charakter der Sowjetunion bestimmen, müssen von daher kritisch befragt werden. Eine fundamentale Behauptung ist, daß die ökonomische Basis der Sowjetunion als verstaatlichte Industrie sozialistisch sei. Das war nicht nur die implizite Voraussetzung der meisten bolschewistischen Theoretiker in den 20er Jahren in der Diskussion um den Aufbau des Sozialismus in der Sowjetunion (18), das ist nicht nur die Prämisse der offiziellen sowjetmarxistischen Legitimationsideologie, sondern auch vieler marxistischer Kritiker der Sowjetunion. Sei es die vorsichtige Kritik am Stalinismus innerhalb der westeuropäischen Kommunistischen Parteien, sei es der Vorwurf der Überzentralisierung und der fehlenden Demokratie durch M. Dobb (19), sei es Medwedews Anklage der mangelnden Demokratisierung von Partei und Staat als Ursache des Stalinismus, oder sei es auch die trotzkistische Beurteilung der Sowjetunion als deformierter Arbeiterstaat (20), immer wird vorausgesetzt, daß die verstaatlichte Industrie sozialistische Vergesellschaftung sei. Man kommt dann nicht nur in die Schwierigkeit, den Stalinismus auf Fehler der Führung, geschichtliche Auswüchse eines Bürokratismus, kapitalistische Relikte etc. zurückzuführen und damit in unmaterialistische Geschichtsschreibung zurückzufallen, sondern macht den zentralen Fehler, verstaatlichte Industrie und staatliche Planung per se für Sozialismus zu erklären und gerade von dem Inhalt dieser Verstaatlichung zu abstrahieren. Auch große Teile der zaristischen Industrie waren verstaatlicht und wurden staatlich geplant, aber niemand würde auf die Idee kommen, dies auch nur für ein Gramm Sozialismus zu halten. In zunehmendem Umfang werden auch in hochkapitalistischen Ländern Betriebe unter staatliche Regie genommen, aber von marxistischen Kritikern ist immer zurecht daran festgehalten worden, daß diese Verstaatlichung auf Basis der kapitalistischen Produktion, auf kapitalistischer Verwertung und Lohnarbeit geschieht. In diesem Sinne ist eine Industrialisierung, die sich auf die Technologie des ausländischen Kapitals und in umfangreichem Maße auf staatliche Despotie stützt, eben auch nicht eine sozialistische Ökonomie, d.h. eine bewußte und freie Vergesellschaftung.

Auch die verbreitete Konzeption, die Sowjetunion sei eine Übergangsgesellschaft zwischen Kapitalismus und Sozialismus, muß kritisch befragt werden.

18 Stellvertretend für die beiden Hauptfraktionen und bei allen ökonomischen und politischen Differenzen: N. Bucharin, Ökonomik der Transformationsperiode, Reinbek 1970 und E. Preobaschenskij, Die Neue Ökonomik, Berlin 1971

19 z.B. in Bettelheim, u.a.: Kritik der Sowjetökonomie, Berlin 1969

20 R. Medwedew: Die Wahrheit ist unsere Stärke, Frankfurt a. M. 1973 
Nehmen wir nur zwei wesentliche Varianten. Mandel (21) versucht, die konkreten Gesellschaftsphänomene der Sowjetunion als Produkt antagonistischer Gesetze von Produktionsweisen, d.h. aus kapitalistischen Muttermalen und schon gebildeten sozialistischen Strukturen zu erklären. Für Bettelheim (22) war die Sowjetunion eine Übergangsgesellschaft, in der mit den ökonomischen Reformen die kapitalistische Produktion, wenn auch in der spezifischen Form einer Staatsbourgeoisie wieder die Oberhand gewonnen hat. Abgesehen von der schon kritisierten Prämisse, verstaatlichte und staatlich geplante Industrie mit Sozialismus gleichzusetzen, wird der Fehler gemacht, die Marxschen Formulierungen der Kritik des Gothaer Programms über die zwei Phasen des Kommunismus als naturnotwendig ablaufende, nach einer sozialistischen Revolution unwiderrufliche Stadien aufzufassen und auf die Sowjetunion zu projizieren. Kein Zweifel, die Vergesellschaftung im Rahmen der kapitalistischen Produktionsweise schafft die Voraussetzungen und ist die Basis ihrer Freisetzung, aber diese soziale Emanzipation muß praktisch verwirklicht werden, sie setzt sich nicht als überhistorische Notwendigkeit durch. Es widerspricht sich also gar nicht, die Oktoberrevolution in ihren proletarischen Tendenzen als sozialistische Revolution zu kennzeichnen und dennoch davon auszugehen, daß mit der Zwangskollektivierung und der forcierten Industrialisierung die Muttermale der sowjetischen Gesellschaft sich in neuer Form durchgesetzt haben. Wenn man von der Konzeption ausgeht, daß die Sowjetunion eine Übergangsgesellschaft zwischen Kapitalismus und Sozialismus ist, setzt man einerseits voraus, daß die zaristische Gesellschaft eine kapitalistische war, und andererseits, daß die sozialistischen Tendenzen lange Zeit wirksam waren oder bis heute wirksam sind. Aber die zaristische Gesellschaft war nie eine rein kapitalistische Gesellschaft, sondern eine durch das Zusammenspiel von feudal-asiatischen und modern-kapitalistischen Elementen in spezifischer Weise kombinierte Gesellschaft, die auch die Transformationsbedingungen der sowjetischen Gesellschaft bestimmten. Also die Muttermale der sowjetischen Gesellschaft sind nicht nur kapitalistische, und es ist von daher falsch, den russischen Thermidor in der Restitution einer rein kapitalistischen Gesellschaft zu erwarten, sondern er besteht in der Wiederherstellung einer über die despotische Staatsgewalt vermittelten, in Abhängigkeit vom Weltmarkt und unter extrem rückständigen Gesellschaftsbedingungen stattfindenden industriellen Entwicklung. Der Stalinismus war allerdings die gesellschaftliche Form, in der die Vergesellschaftung der Arbeit durchgeführt wurde - ohne ihn hätte Rußland wie gesagt das Schicksal der heute unterentwickelten Länder erwartet -, und damit sind die materiellen Voraussetzungen für einen erneuten Versuch der sozialen Emanzipation ganz anders als vordem geschaffen. Insofern ist die Sowjetunion auch Übergangsgesellschaft, aber nur in dem allgemeinen Sinne, wie die kapitalistische Industrialisierung Übergang zur Produktionsweise der freien und assoziierten

21 E. Mandel: Zehn Thesen zur sozial-ökonomischen Gesetzmäßigkeit der Übergangsgesellschaft zwischen Kapitalismus und Sozialismus, in: Hennicke (Hg.), Probleme des Sozialismus und der Übergangsgesellschaften, Frankfurt a.M. 1973 C. Bettelheim; Ökonomisches Kalkül und Eigentumsformen, Berlin 1972 
Produzenten ist; im Fall der Sowjetunion also eine Industrialisierung unter extrem rückständigen Voraussetzungen und deshalb auch unter einer besonders despotischen Staatsform, die sich in dem Maße liberalisiert, wie die Rückständigkeit wunden wird. Aber diese Vergesellschaftung der Arbeit, nur weil sie in exzessiver Staatlichkeit vollzogen wird, als per se sozialistisch oder als per se im Übergang zum Sozialismus zu begreifen, hat genausowenig Sinn, wie die Vergesellschaftung der Arbeit unter rein kapitalistischen Bedingungen als solche für Sozialismus zu halten, denn sie ist nur die Basis der sozialen Emanzipation, nicht aber diese selbst.

Zwei weitere Konzeptionen zeigen die Schwierigkeit, die Gesellschaftsstruktur der Sowjetunion einfach als Definition fassen zu wollen. So wurde in Anlehnung an Lenins Charakterisierung der Sowjetunion während der NÖP als Staatskapitalismus dieser Begriff auf die gesamte sowjetische Entwicklung übertragen (23). Dieser Staatskapitalismus wird als spezifisches Übergangsstadium zwischen Kapitalismus und Sozialismus - getreu der Bucharinschen und Leninschen Stadientheorie angesiedelt. Aber auf diese Weise werden die spezifischen Unterschiede zwischen den verschiedenen Phasen der Sowjetunion: der NÖP, der Stalinschen Industrialisierung und der ökonomischen Reformen verwischt und vor allem übersehen, daß die Staatsmacht der Sowjetunion nicht das Produkt einer Gesellschaft ist, die höher als der „Privatkapitalismus“ entwickelt ist, sondern das Produkt einer Industrialisierung unter relativ rïckständigen Gesellschaftsbedingungen. Noch problematischer ist die Definition der Sowjetunion als etatistische oder bürokratisch-kollektivistische Gesellschaft (24). Diese Definition erfaßt wohl die Erscheinung einer extrem ausgeprägten Staatlichkeit, vergißt aber den Inhalt und die Ursachen der Staatlichkeit als Wesen der Sache zu nehmen. Genausogut könnte der Staat Peters des Großen als Etatismus oder bürokratischer Kollektivismus gekennzeichnet werden, ohne daß man dem spezifischen Charakter dieser Staatsmacht einen Schritt näher gekommen wäre.

Die Absicht war, nicht eine eigene feststehende Definition des gesellschaftlichen Charakters der Sowjetunion anzubieten, sondern es sollte auf Basis der methodischen Prämisse, daß die Entwicklung der zaristischen und sowjetischen Gesellschaft nur im Kontext des Weltmarkts zu analysieren ist, und auf Basis der hierfür relevanten Resultate der Suttonschen Untersuchung gezeigt werden, wie eine materialistische Erklärung der Entwicklung der sowjetischen Gesellschaft angegangen werden kann. Dieser Interpretationsrahmen hat allerdings zur Voraussetzung, nicht von postulierten überhistorischen Abstraktionen auszugehen, die immer die Gefahr der Ideologisierung in sich tragen, sondern die geschichtliche Entwicklung der Sowjetunion in ihren konkreten Zusammenhängen, d.h. von ihren inneren und äußeren Bedingungen her zu bestimmen. Die Resultate der hierzu bestehenden historischen Untersuchungen geben allerdings Anlaß, bisher umstandslos vorausgesetzte Prämissen in der marxistischen Diskussion über den gesellschaftlichen Charakter der Sowjetunion in Frage zu stellen.

24 A. Carlo; a.a.O. und Eggert, a.a.O. 